\title{
Condições Teórico-Práticas das Ciências da Saúde no Brasil 3
}

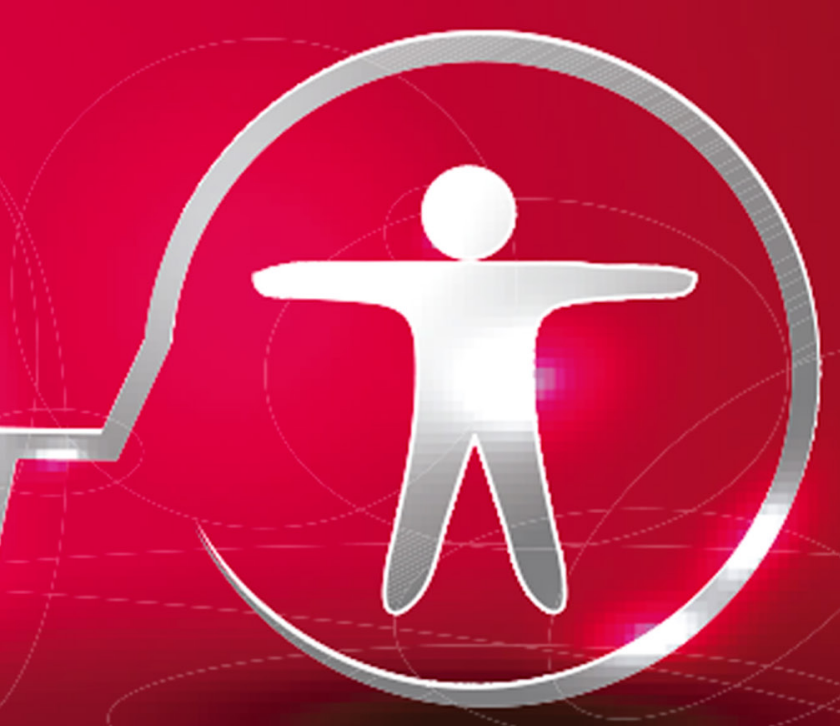

Luis Henrique Almeida Castro

(Organizador)

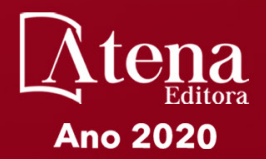




\section{Condições Teórico-Práticas das Ciências da Saúde no Brasil 3}

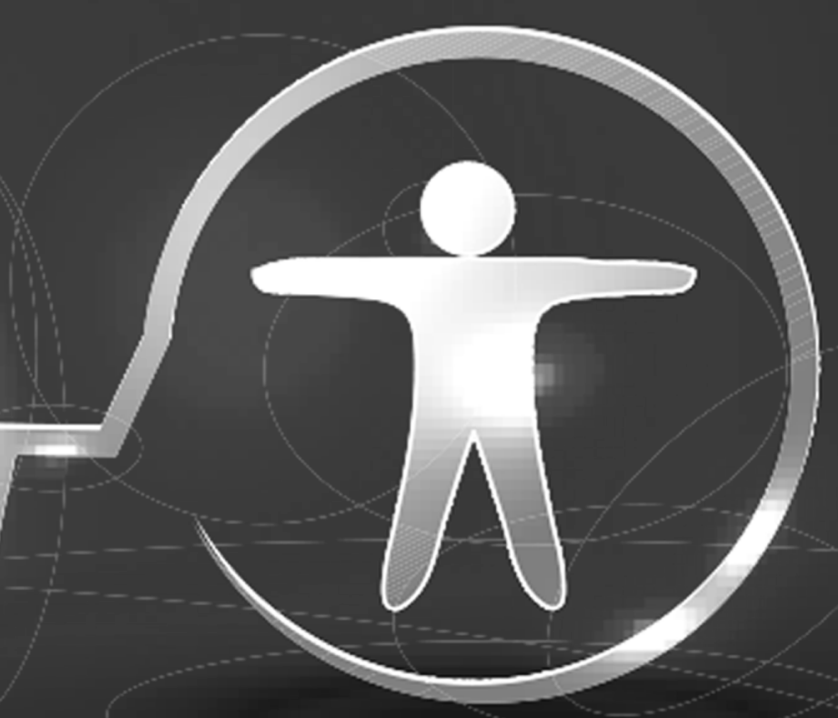

Luis Henrique Almeida Castro

(Organizador)

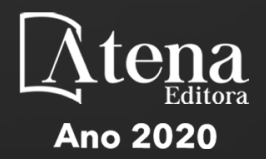




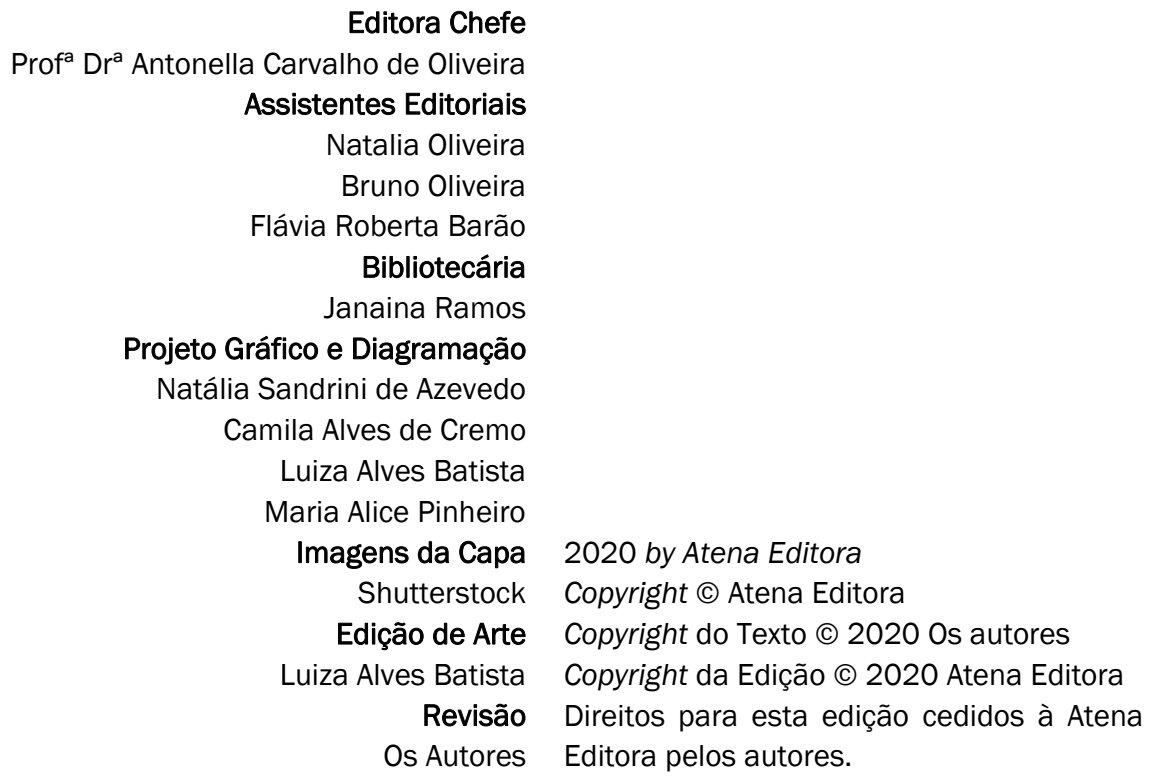

Todo o conteúdo deste livro está licenciado sob uma Licença de Atribuição Creative Commons. Atribuição-Não-ComercialNãoDerivativos 4.0 Internacional (CC BY-NC-ND 4.0).

O conteúdo dos artigos e seus dados em sua forma, correção e confiabilidade são de responsabilidade exclusiva dos autores, inclusive não representam necessariamente a posição oficial da Atena Editora. Permitido o download da obra e o compartilhamento desde que sejam atribuídos créditos aos autores, mas sem a possibilidade de alterá-la de nenhuma forma ou utilizá-la para fins comerciais.

Todos os manuscritos foram previamente submetidos à avaliação cega pelos pares, membros do Conselho Editorial desta Editora, tendo sido aprovados para a publicação.

A Atena Editora é comprometida em garantir a integridade editorial em todas as etapas do processo de publicação. Situações suspeitas de má conduta científica serão investigadas sob o mais alto padrão de rigor acadêmico e ético.

\section{Conselho Editorial}

Ciências Humanas e Sociais Aplicadas

Prof. Dr. Alexandre Jose Schumacher - Instituto Federal de Educação, Ciência e Tecnologia do Paraná

Prof. Dr. Américo Junior Nunes da Silva - Universidade do Estado da Bahia

Prof. Dr. Antonio Carlos Frasson - Universidade Tecnológica Federal do Paraná

Prof. Dr. Antonio Gasparetto Júnior - Instituto Federal do Sudeste de Minas Gerais

Prof. Dr. Antonio Isidro-Filho - Universidade de Brasília

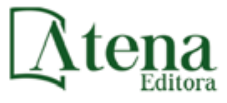

Ano 2020 
Prof. Dr. Carlos Antonio de Souza Moraes - Universidade Federal Fluminense

Prof $^{a}$ Dr $^{\text {a }}$ Cristina Gaio - Universidade de Lisboa

Prof. Dr. Daniel Richard Sant'Ana - Universidade de Brasília

Prof. Dr. Deyvison de Lima Oliveira - Universidade Federal de Rondônia

Prof ${ }^{a}$ Dr $^{\text {a }}$ Dilma Antunes Silva - Universidade Federal de São Paulo

Prof. Dr. Edvaldo Antunes de Farias - Universidade Estácio de Sá

Prof. Dr. Elson Ferreira Costa - Universidade do Estado do Pará

Prof. Dr. Eloi Martins Senhora - Universidade Federal de Roraima

Prof. Dr. Gustavo Henrique Cepolini Ferreira - Universidade Estadual de Montes Claros

Prof $^{a} \mathrm{Dr}^{\mathrm{a}}$ Ivone Goulart Lopes - Istituto Internazionele delle Figlie de Maria Ausiliatrice

Prof. Dr. Jadson Correia de Oliveira - Universidade Católica do Salvador

Prof. Dr. Julio Candido de Meirelles Junior - Universidade Federal Fluminense

Prof $^{a} \mathrm{Dr}^{\mathrm{a}}$ Lina Maria Gonçalves - Universidade Federal do Tocantins

Prof. Dr. Luis Ricardo Fernandes da Costa - Universidade Estadual de Montes Claros

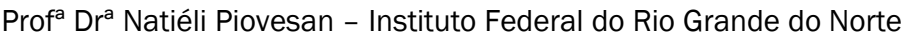

Prof. Dr. Marcelo Pereira da Silva - Pontifícia Universidade Católica de Campinas

Prof $^{a}$ Dr $^{a}$ Maria Luzia da Silva Santana - Universidade Federal de Mato Grosso do Sul

Prof $^{a}$ Dr $^{\text {a }}$ Paola Andressa Scortegagna - Universidade Estadual de Ponta Grossa

Prof $^{a}$ Dr $^{a}$ Rita de Cássia da Silva Oliveira - Universidade Estadual de Ponta Grossa

Prof. Dr. Rui Maia Diamantino - Universidade Salvador

Prof. Dr. Urandi João Rodrigues Junior - Universidade Federal do Oeste do Pará

Prof $^{a}$ Dr $^{a}$ Vanessa Bordin Viera - Universidade Federal de Campina Grande

Prof. Dr. William Cleber Domingues Silva - Universidade Federal Rural do Rio de Janeiro

Prof. Dr. Willian Douglas Guilherme - Universidade Federal do Tocantins

\section{Ciências Agrárias e Multidisciplinar}

Prof. Dr. Alexandre Igor Azevedo Pereira - Instituto Federal Goiano

Prof $^{\mathrm{a}} \mathrm{Dr}^{\mathrm{a}}$ Carla Cristina Bauermann Brasil - Universidade Federal de Santa Maria

Prof. Dr. Antonio Pasqualetto - Pontifícia Universidade Católica de Goiás

Prof. Dr. Cleberton Correia Santos - Universidade Federal da Grande Dourados

Prof $^{a}$ Dr $^{a}$ Daiane Garabeli Trojan - Universidade Norte do Paraná

Prof ${ }^{a}$ Dr $^{a}$ Diocléa Almeida Seabra Silva - Universidade Federal Rural da Amazônia

Prof. Dr. Écio Souza Diniz - Universidade Federal de Viçosa

Prof. Dr. Fábio Steiner - Universidade Estadual de Mato Grosso do Sul

Prof. Dr. Fágner Cavalcante Patrocínio dos Santos - Universidade Federal do Ceará

Prof ${ }^{a}$ Dr $^{a}$ Girlene Santos de Souza - Universidade Federal do Recôncavo da Bahia

Prof. Dr. Jael Soares Batista - Universidade Federal Rural do Semi-Árido

Prof. Dr. Júlio César Ribeiro - Universidade Federal Rural do Rio de Janeiro

Prof $^{a}$ Dr $^{a}$ Lina Raquel Santos Araújo - Universidade Estadual do Ceará

Prof. Dr. Pedro Manuel Villa - Universidade Federal de Viçosa

Prof $^{a}$ Dr $^{a}$ Raissa Rachel Salustriano da Silva Matos - Universidade Federal do Maranhão

Prof. Dr. Ronilson Freitas de Souza - Universidade do Estado do Pará

Prof $^{\mathrm{a}} \mathrm{Dr}^{\mathrm{a}}$ Talita de Santos Matos - Universidade Federal Rural do Rio de Janeiro

Prof. Dr. Tiago da Silva Teófilo - Universidade Federal Rural do Semi-Árido

Prof. Dr. Valdemar Antonio Paffaro Junior - Universidade Federal de Alfenas

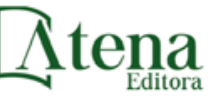

Ano 2020 


\section{Ciências Biológicas e da Saúde}

Prof. Dr. André Ribeiro da Silva - Universidade de Brasília

Prof $^{\mathrm{a}} \mathrm{Dr}^{\mathrm{a}}$ Anelise Levay Murari - Universidade Federal de Pelotas

Prof. Dr. Benedito Rodrigues da Silva Neto - Universidade Federal de Goiás

Prof $^{a}$ Dr $^{a}$ Débora Luana Ribeiro Pessoa - Universidade Federal do Maranhão

Prof. Dr. Douglas Siqueira de Almeida Chaves -Universidade Federal Rural do Rio de Janeiro

Prof. Dr. Edson da Silva - Universidade Federal dos Vales do Jequitinhonha e Mucuri

Prof $^{\mathrm{a}} \mathrm{Dr}^{\mathrm{a}}$ Eleuza Rodrigues Machado - Faculdade Anhanguera de Brasília

Prof $^{a}$ Dr $^{\mathrm{a}}$ Elane Schwinden Prudêncio - Universidade Federal de Santa Catarina

Prof $^{\mathrm{a}}$ Dr $^{\mathrm{a}}$ Eysler Gonçalves Maia Brasil - Universidade da Integração Internacional da Lusofonia

Afro-Brasileira

Prof. Dr. Ferlando Lima Santos - Universidade Federal do Recôncavo da Bahia

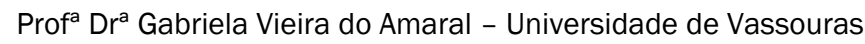

Prof. Dr. Gianfábio Pimentel Franco - Universidade Federal de Santa Maria

Prof. Dr. Helio Franklin Rodrigues de Almeida - Universidade Federal de Rondônia

Prof $^{a}$ Dr $^{\mathrm{a}}$ lara Lúcia Tescarollo - Universidade São Francisco

Prof. Dr. Igor Luiz Vieira de Lima Santos - Universidade Federal de Campina Grande

Prof. Dr. Jefferson Thiago Souza - Universidade Estadual do Ceará

Prof. Dr. Jesus Rodrigues Lemos - Universidade Federal do Piauí

Prof. Dr. Jônatas de França Barros - Universidade Federal do Rio Grande do Norte

Prof. Dr. José Max Barbosa de Oliveira Junior - Universidade Federal do Oeste do Pará

Prof. Dr. Luís Paulo Souza e Souza - Universidade Federal do Amazonas

Prof $^{\mathrm{a}} \mathrm{Dr}^{\mathrm{a}}$ Magnólia de Araújo Campos - Universidade Federal de Campina Grande

Prof. Dr. Marcus Fernando da Silva Praxedes - Universidade Federal do Recôncavo da Bahia

Prof $^{\mathrm{a}} \mathrm{Dr}^{\mathrm{a}}$ Maria Tatiane Gonçalves Sá - Universidade do Estado do Pará

Prof $^{a}$ Dr $^{\text {a }}$ Mylena Andréa Oliveira Torres - Universidade Ceuma

Prof $^{\mathrm{a}}$ Dr $^{\mathrm{a}}$ Natiéli Piovesan - Instituto Federacl do Rio Grande do Norte

Prof. Dr. Paulo Inada - Universidade Estadual de Maringá

Prof. Dr. Rafael Henrique Silva - Hospital Universitário da Universidade Federal da Grande Dourados

Prof $^{a}$ Dr $^{\mathrm{a}}$ Regiane Luz Carvalho - Centro Universitário das Faculdades Associadas de Ensino

Prof $^{a}$ Dr $^{a}$ Renata Mendes de Freitas - Universidade Federal de Juiz de Fora

Prof $^{a}$ Dr $^{a}$ Vanessa Lima Gonçalves - Universidade Estadual de Ponta Grossa

Prof $^{\mathrm{a}} \mathrm{Dr}^{\mathrm{a}}$ Vanessa Bordin Viera - Universidade Federal de Campina Grande

\section{Ciências Exatas e da Terra e Engenharias}

Prof. Dr. Adélio Alcino Sampaio Castro Machado - Universidade do Porto

Prof. Dr. Carlos Eduardo Sanches de Andrade - Universidade Federal de Goiás

Prof $^{\mathrm{a}} \mathrm{Dr}^{\mathrm{a}}$ Carmen Lúcia Voigt - Universidade Norte do Paraná

Prof. Dr. Douglas Gonçalves da Silva - Universidade Estadual do Sudoeste da Bahia

Prof. Dr. Eloi Rufato Junior - Universidade Tecnológica Federal do Paraná

Prof $^{a}$ Dr $^{a}$ Érica de Melo Azevedo - Instituto Federal do Rio de Janeiro

Prof. Dr. Fabrício Menezes Ramos - Instituto Federal do Pará

Prof $^{a}$ Dra. Jéssica Verger Nardeli - Universidade Estadual Paulista Júlio de Mesquita Filho

Prof. Dr. Juliano Carlo Rufino de Freitas - Universidade Federal de Campina Grande

Prof $^{\mathrm{a}} \mathrm{Dr}^{\mathrm{a}}$ Luciana do Nascimento Mendes - Instituto Federal de Educação, Ciência e Tecnologia do Rio Grande do Norte

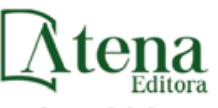


Prof. Dr. Marcelo Marques - Universidade Estadual de Maringá

Prof $^{a} \operatorname{Dr}^{\mathrm{a}}$ Neiva Maria de Almeida - Universidade Federal da Paraíba

Prof $^{a}$ Dr $^{a}$ Natiéli Piovesan - Instituto Federal do Rio Grande do Norte

Prof ${ }^{a}$ Dr $^{\text {a }}$ Priscila Tessmer Scaglioni - Universidade Federal de Pelotas

Prof. Dr. Takeshy Tachizawa - Faculdade de Campo Limpo Paulista

\section{Linguística, Letras e Artes}

Prof $^{a}$ Dr $^{a}$ Adriana Demite Stephani - Universidade Federal do Tocantins

Prof $^{a}$ Dr $^{a}$ Angeli Rose do Nascimento - Universidade Federal do Estado do Rio de Janeiro

Prof $^{a}$ Dra $^{\text {a }}$ Carolina Fernandes da Silva Mandaji - Universidade Tecnológica Federal do Paraná

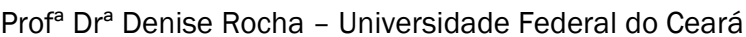

Prof. Dr. Fabiano Tadeu Grazioli - Universidade Regional Integrada do Alto Uruguai e das Missões

Prof. Dr. Gilmei Fleck - Universidade Estadual do Oeste do Paraná

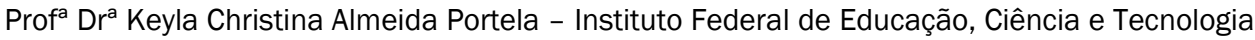
do Paraná

Prof $^{a}$ Dra $^{a}$ Miranilde Oliveira Neves - Instituto de Educação, Ciência e Tecnologia do Pará

Prof $^{a}$ Dr $^{\text {a }}$ Sandra Regina Gardacho Pietrobon - Universidade Estadual do Centro-Oeste

Prof $^{a}$ Dr $^{\text {a }}$ Sheila Marta Carregosa Rocha - Universidade do Estado da Bahia

\section{Conselho Técnico Científico}

Prof. Me. Abrãao Carvalho Nogueira - Universidade Federal do Espírito Santo

Prof. Me. Adalberto Zorzo - Centro Estadual de Educação Tecnológica Paula Souza

Prof. Dr. Adaylson Wagner Sousa de Vasconcelos - Ordem dos Advogados do Brasil/Seccional

Paraíba

Prof. Dr. Adilson Tadeu Basquerote Silva - Universidade para o Desenvolvimento do Alto Vale do Itajaí

Prof. Me. Alexsandro Teixeira Ribeiro - Centro Universitário Internacional

Prof. Me. André Flávio Gonçalves Silva - Universidade Federal do Maranhão

Prof ${ }^{a}$ Ma. Andréa Cristina Marques de Araújo - Universidade Fernando Pessoa

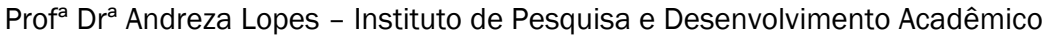

Prof $^{a}$ Dr $^{\text {a }}$ Andrezza Miguel da Silva - Faculdade da Amazônia

Prof ${ }^{a}$ Ma. Anelisa Mota Gregoleti - Universidade Estadual de Maringá

Prof $^{a}$ Ma. Anne Karynne da Silva Barbosa - Universidade Federal do Maranhão

Prof. Dr. Antonio Hot Pereira de Faria - Polícia Militar de Minas Gerais

Prof. Me. Armando Dias Duarte - Universidade Federal de Pernambuco

Prof $^{\mathrm{a}}$ Ma. Bianca Camargo Martins - UniCesumar

Prof $^{a}$ Ma. Carolina Shimomura Nanya - Universidade Federal de São Carlos

Prof. Me. Carlos Antônio dos Santos - Universidade Federal Rural do Rio de Janeiro

Prof. Ma. Cláudia de Araújo Marques - Faculdade de Música do Espírito Santo

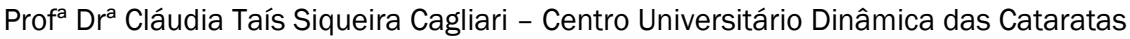

Prof. Me. Clécio Danilo Dias da Silva - Universidade Federal do Rio Grande do Norte

Prof. Me. Daniel da Silva Miranda - Universidade Federal do Pará

Prof $^{a}$ Ma. Daniela da Silva Rodrigues - Universidade de Brasília

Prof $^{a}$ Ma. Daniela Remião de Macedo - Universidade de Lisboa

Prof $^{a}$ Ma. Dayane de Melo Barros - Universidade Federal de Pernambuco

Prof. Me. Douglas Santos Mezacas - Universidade Estadual de Goiás

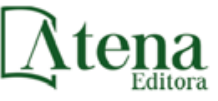

Ano 2020 
Prof. Me. Edevaldo de Castro Monteiro - Embrapa Agrobiologia

Prof. Me. Eduardo Gomes de Oliveira - Faculdades Unificadas Doctum de Cataguases

Prof. Me. Eduardo Henrique Ferreira - Faculdade Pitágoras de Londrina

Prof. Dr. Edwaldo Costa - Marinha do Brasil

Prof. Me. Eliel Constantino da Silva - Universidade Estadual Paulista Júlio de Mesquita

Prof. Me. Ernane Rosa Martins - Instituto Federal de Educação, Ciência e Tecnologia de Goiás

Prof. Me. Euvaldo de Sousa Costa Junior - Prefeitura Municipal de São João do Piauí

Prof $^{a}$ Ma. Fabiana Coelho Couto Rocha Corrêa - Centro Universitário Estácio Juiz de Fora

Prof. Me. Felipe da Costa Negrão - Universidade Federal do Amazonas

Prof ${ }^{\mathrm{a}} \mathrm{Dr}^{\mathrm{a}}$ Germana Ponce de Leon Ramírez - Centro Universitário Adventista de São Paulo

Prof. Me. Gevair Campos - Instituto Mineiro de Agropecuária

Prof. Me. Givanildo de Oliveira Santos - Secretaria da Educação de Goiás

Prof. Dr. Guilherme Renato Gomes - Universidade Norte do ParanáProf. Me. Gustavo Krahl Universidade do Oeste de Santa Catarina

Prof. Me. Helton Rangel Coutinho Junior - Tribunal de Justiça do Estado do Rio de Janeiro

Prof $^{a}$ Ma. Isabelle Cerqueira Sousa - Universidade de Fortaleza

Prof ${ }^{a}$ Ma. Jaqueline Oliveira Rezende - Universidade Federal de Uberlândia

Prof. Me. Javier Antonio Albornoz - University of Miami and Miami Dade College

Prof. Me. Jhonatan da Silva Lima - Universidade Federal do Pará

Prof. Dr. José Carlos da Silva Mendes - Instituto de Psicologia Cognitiva, Desenvolvimento Humano e Social

Prof. Me. Jose Elyton Batista dos Santos - Universidade Federal de Sergipe

Prof. Me. José Luiz Leonardo de Araujo Pimenta - Instituto Nacional de Investigación Agropecuaria Uruguay

Prof. Me. José Messias Ribeiro Júnior - Instituto Federal de Educação Tecnológica de Pernambuco

Prof $^{a}$ Dr $^{a}$ Juliana Santana de Curcio - Universidade Federal de Goiás

Prof ${ }^{a}$ Ma. Juliana Thaisa Rodrigues Pacheco - Universidade Estadual de Ponta Grossa

Prof $^{a}$ Dr $^{a}$ Kamilly Souza do Vale - Núcleo de Pesquisas Fenomenológicas/UFPA

Prof. Dr. Kárpio Márcio de Siqueira - Universidade do Estado da Bahia

Prof $^{a}$ Dr $^{a}$ Karina de Araújo Dias - Prefeitura Municipal de Florianópolis

Prof. Dr. Lázaro Castro Silva Nascimento - Laboratório de Fenomenologia \& Subjetividade/UFPR

Prof. Me. Leonardo Tullio - Universidade Estadual de Ponta Grossa

Prof $^{a}$ Ma. Lilian Coelho de Freitas - Instituto Federal do Pará

Prof ${ }^{a}$ Ma. Liliani Aparecida Sereno Fontes de Medeiros - Consórcio CEDERJ

Prof $^{a}$ Dr $^{a}$ Lívia do Carmo Silva - Universidade Federal de Goiás

Prof. Dr. Lucio Marques Vieira Souza - Secretaria de Estado da Educação, do Esporte e da Cultura de Sergipe

Prof. Me. Luis Henrique Almeida Castro - Universidade Federal da Grande Dourados

Prof. Dr. Luan Vinicius Bernardelli - Universidade Estadual do Paraná

Prof. Dr. Michel da Costa - Universidade Metropolitana de Santos

Prof. Dr. Marcelo Máximo Purificação - Fundação Integrada Municipal de Ensino Superior

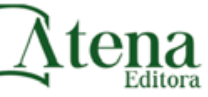

Ano 2020 
Prof. Me. Marcos Aurelio Alves e Silva - Instituto Federal de Educação, Ciência e Tecnologia de São Paulo

Prof ${ }^{a}$ Ma. Maria Elanny Damasceno Silva - Universidade Federal do Ceará

Prof ${ }^{a}$ Ma. Marileila Marques Toledo - Universidade Federal dos Vales do Jequitinhonha e Mucuri

Prof. Me. Ricardo Sérgio da Silva - Universidade Federal de Pernambuco

Prof ${ }^{a}$ Ma. Renata Luciane Polsaque Young Blood - UniSecal

Prof. Me. Robson Lucas Soares da Silva - Universidade Federal da Paraíba

Prof. Me. Sebastião André Barbosa Junior - Universidade Federal Rural de Pernambuco

Prof $^{a}$ Ma. Silene Ribeiro Miranda Barbosa - Consultoria Brasileira de Ensino, Pesquisa e Extensão

Prof $^{a}$ Ma. Solange Aparecida de Souza Monteiro - Instituto Federal de São Paulo

Prof. Me. Tallys Newton Fernandes de Matos - Faculdade Regional Jaguaribana

Prof $^{a}$ Ma. Thatianny Jasmine Castro Martins de Carvalho - Universidade Federal do Piauí

Prof. Me. Tiago Silvio Dedoné - Colégio ECEL Positivo

Prof. Dr. Welleson Feitosa Gazel - Universidade Paulista 
Editora Chefe: Prof $^{\mathrm{a}} \mathrm{Dr}^{\mathrm{a}}$ Antonella Carvalho de Oliveira

Bibliotecária: Janaina Ramos

Diagramação: Luiza Alves Batista

Correção: Giovanna Sandrini de Azevedo

Edição de Arte: Luiza Alves Batista

Revisão: Os Autores

Organizador: Luis Henrique Almeida Castro

Dados Internacionais de Catalogação na Publicação (CIP)

C745 Condições teórico-práticas das ciências da saúde no Brasil 3 / Organizador Luis Henrique Almeida Castro. - Ponta Grossa - PR: Atena, 2020.

Formato: PDF

Requisitos de sistema: Adobe Acrobat Reader Modo de acesso: World Wide Web

Inclui bibliografia

ISBN 978-65-5706-639-3

DOI 10.22533/at.ed.393200312

1. Saúde. 2. Ciências. I. Castro, Luis Henrique Almeida (Organizador). II. Título.

CDD 613

Elaborado por Bibliotecária Janaina Ramos - CRB-8/9166

Atena Editora

Ponta Grossa - Paraná - Brasil Telefone: +55 (42) 3323-5493 www.atenaeditora.com.br contato@atenaeditora.com.br

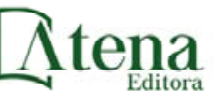

Ano 2020 


\section{DECLARAÇÃO DOS AUTORES}

Os autores desta obra: 1. Atestam não possuir qualquer interesse comercial que constitua um conflito de interesses em relação ao artigo científico publicado; 2. Declaram que participaram ativamente da construção dos respectivos manuscritos, preferencialmente na: a) Concepção do estudo, e/ou aquisição de dados, e/ou análise e interpretação de dados; b) Elaboração do artigo ou revisão com vistas a tornar o material intelectualmente relevante; c) Aprovação final do manuscrito para submissão; 3. Certificam que os artigos científicos publicados estão completamente isentos de dados e/ou resultados fraudulentos. 


\section{APRESENTAÇÃO}

A Atena Editora traz ao leitor na obra "Condições Teórico-Práticas das Ciências da Saúde no Brasil” 69 estudos científicos que investigaram, com uma abordagem plural, o panorama nacional acerca dos desafios que a ciência e a academia científica enfrentam ante a saúde pública.

Os textos foram compilados em três volumes, cada qual com seu eixo temático, respectivamente: "População Brasileira \& Saúde Pública", que traz ao leitor estudos que investigaram algumas das principais patologias que compõe o quadro epidemiológico no Brasil atual; "Atuação Profissional em Saúde" que, por sua vez, é composto por artigos que revisam o papel do profissional de saúde seja em sua formação acadêmica, seja em sua atuação clínica; e, "Cuidado Integrado e Terapêutico", volume que apresenta, discute e/ou propõe opções de terapia em saúde coletiva e individual com foco nos aspectos biopsicossociais que permeiam o cotidiano da saúde no país.

Almeja-se que a leitura deste e-book possa incentivar o desenvolvimento de estratégias de atuação coletiva, educacional e de inclusão social de modo a subsidiar, na esfera do condicionamento teórico e prático, a continuidade da produção científica brasileira.

Boa leitura!

Luis Henrique Almeida Castro 


\section{SUMÁRIO}

\section{CUIDADO INTEGRADO E TERAPÊUTICO}

CAPÍTULO 1.

AÇÃO CRIANÇA FELIZ: INTERDISCIPLINARIDADE E MULTIDISCIPLINARIDADE EM CRIANÇAS HOSPITALIZADAS

Najara Paiva dos Santos

Izadora Larissa Cei Lima

Thayse Kelly da Silva Martino

Keniellry Daris Pinheiro

Francisca Maynara de Aguiar Bastos

João Paulo Lima da Silva

Jefferson Michael Barros do Rosário

Lucas Deyver da Paixão Lima

Philip Daniel Toth

Felipe Souza Nascimento

Fernando de Souza Lima

Alana Thais do Rosário Ribeiro

DOI 10.22533/at.ed.3932003121

CAPÍTULO 2

APLICAÇÃO DE GENOGRAMA EM FAMÍLIAS COM CASOS DE HANSENÍASE NO LESTE DE MINAS GERAIS PARA AUXÍLIO NA CARACTERIZAÇÃO CLÍNICA-SÓCIODEMOGRÁFICA

Lucia Alves de Oliveira Fraga

Andre de Souza Otaviano

Regiani Lucia Riani

Patricia Zandim

Cibele Velloso-Rodrigues

Rodrigo de Paiva Souza

Márcio Luís Moreira de Souza

Gulnara Borja Cabrera

Pauline Martins Leite

Pedro Henrique Ferreira Marçal

Lorena Bruna Pereira de Oliveira

Rafael Silva Gama

Thalisson Artur Ribero Gomides

Érica Barbosa Magueta

Maria Aparecida Grossi

Jessica Fairley

DOI 10.22533/at.ed.3932003122

CAPÍTULO 3

ASSOCIAÇÃO DO USO DAS PRÁTICAS INTEGRATIVAS E CUIDADOS PALIATIVOS NA QUALIDADE DE VIDA DOS PACIENTES COM AS PRINCIPAIS DOENÇAS NEUROLÓGICAS

Michel Rodrigues de Carvalho Perroti 
DOI 10.22533/at.ed.3932003123

CAPÍTULO 4

AVALIAÇÃO DOS NÍVEIS DE EXPRESSÃO DO MIRNA-155 NAS NEOPLASIAS CERVICAIS INTRAEPITELIAIS DE ALTO E BAIXO GRAU: PROSPECÇÃO DE UM BIOMARCADOR DIAGNÓSTICO MOLECULAR

Alina Laís Almeida de Farias Fernandes

Daline Dias dos Santos

Jose Aníbal Matamoros

Eliane Campos Coimbra

DOI 10.22533/at.ed.3932003124

CAPÍTULO 5

COMPREENSÃO DAS MÃES SOBRE O ALEITAMENTO MATERNO E SUA RELAÇÃO COM O TIPO DE PARTO

Ana Paula Desplanches dos Santos

Cristina Ide Fujinaga

Maria Eduarda Mendes Fernandes

Cíntia da Conceição Costa

Paula Maria Pankiw

Cleomara Mocelin Salla

Caroline Gianna da Silva

DOI 10.22533/at.ed.3932003125

CAPÍTULO 6 . 54

CUIDADO FARMACÊUTICO COMO CONTRIBUIÇÃO PARA O FORTALECIMENTO DA ATENÇÃO PRIMÁRIA À SAÚDE

Lohanne Elis Cordeiro Paz

Arcelio Benetoli

Ana Paula Veber

Daniele Priscila da Silva Fardin Assunção

Bruno Rodrigo Minozzo

Gerusa Clazer Halila Possagno

DOI 10.22533/at.ed.3932003126

CAPÍTULO 7. 66

DOR E FUNCIONALIDADE EM IDOSOS COM E SEM HISTÓRICO DE ACIDENTE VASCULAR ENCEFÁLICO

Millena Euzébio da Silva

Vitória Araújo de Paiva

Tiago Tsunoda Del Antonio

Joyce Karla Machado da Silva

Camila Costa de Araujo

DOI 10.22533/at.ed.3932003127 
EDUCAÇÃO EM SAÚDE: CONTINUIDADE NA ASSISTÊNCIA AO RECÉM-NASCIDO PARA ALTA HOSPITALAR EM UNIDADE DE TERAPIA INTENSIVA NEONATAL

Flávia Domingues

Raquel Aparecida de Oliveira

DOI 10.22533/at.ed.3932003128

CAPÍTULO 9 90

EFEITOS DA ACUPUNTURA NO SISTEMA AUDITIVO: REVISÃO BIBLIOGRÁFICA

Elias Victor Figueiredo dos Santos

Carla Karine Figueiredo Lopes

Jadden Rúbia Lima Costa

Maryangela Godinho Pereira Bena

Maria Bernardete Barros Figueiredo

Bruna Katarine Beserra Paz

DOI 10.22533/at.ed.3932003129

CAPÍTULO 10

EFEITOS DE UMA SESSÃO AGUDA DE EXERCÍCIO FÍSICO AERÓBICO SOBRE MARCADORES DE INFLAMAÇÃO E BIOMARCADORES DE FUNÇÃO RENAL

Walter Pereira Pinto

Rafael Andrade Rezende

Armando Morales Júnior

Luiz Phellipe Dell Aquila

Caren Cristina Grabulosa

Rosilene Motta Elias

Taís Tinucci

Maria Aparecida Dalboni

DOI 10.22533/at.ed.39320031210

CAPÍTULO 11

EFICÁCIA DE AÇÕES INTEGRADAS NA REDUÇÃO DA INCIDÊNCIA DE ARBOVIROSES EM UM MUNICÍPIO DO INTERIOR DO CEARÁ

Niciane Bandeira Pessoa Marinho

Francisco Almeida Rocha

Carlecy Rodrigues de Menezes

Lourdes Ramayanne Correia Montenegro

DOI 10.22533/at.ed.39320031211

CAPÍTULO 12 115

ÉSTER DERIVADO DO ÁCIDO GRAXO 18-METIL EICOSANÓICO PARA A REPOSIÇÃO DA BARREIRA LIPÍDICA NATURAL DO CABELO DANIFICADO

Alexandra Macedo Wendler

Fabrício A. de Sousa

Alaor Pereira Lino

DOI 10.22533/at.ed.39320031212 
FOTOEXPOSIÇÃO: EFEITOS DO USO DO LASER DE BAIXA FREQUÊNCIA EM TECIDOS E LINHAGENS DE FIBROBLASTOS (UMA MINIREVISÃO)

Moisés Henrique Mastella

Melissa Gewehr

Fernanda Barbisan

Margrid Beuter

Ivana Beatrice Mânica da Cruz

Bárbara Osmarin Turra

Danieli Monteiro Pillar

Isabel Roggia

Daíse Raquel Maldaner

Marta Maria Medeiros Frescura Duarte

DOI 10.22533/at.ed.39320031213

CAPÍTULO 14

GASTOS COM SERVIÇOS HOSPITALARES RELACIONADOS AOS TRANSTORNOS DE HUMOR: UMA COMPARAÇÃO ENTRE O BRASIL E O ESTADO DE GOIÁS EM 2019

Maria Vitória da Silva Paula Cirilo

Glaucia Borges Dantas

Anna de Paula Freitas Borges

Juliana Beatriz Souza de Freitas

Bárbara de Oliveira Arantes

Samyla Coutinho Paniago

Kamylla Lohannye Fonseca e Silva

Marco Alejandro Menacho Herbas

Anita Abreu de Carvalho

Carlos Hiury Holanda Silva

Karolina de Souza Cardoso

Cristhiano Chiovato Abdala

DOI 10.22533/at.ed.39320031214

CAPÍTULO 15 147

GASTOS COM SERVICYOS HOSPITALARES RELACIONADOS AOS TRANSTORNOS MENTAIS E COMPORTAMENTAIS DEVIDO AO USO DE SUBSTÂNCIAS PSICOATIVAS NO BRASIL: UMA COMPARAÇÃO ENTRE OS ANOS DE 2009 A 2019

Maria Vitória da Silva Paula Cirilo

Glaucia Borges Dantas

Juliana Beatriz Souza de Freitas

Bárbara de Oliveira Arantes

Giane Hayasaki Vieira

Samyla Coutinho Paniago

Kamylla Lohannye Fonseca e Silva

Kristen Guilarducci Laureano

Marco Alejandro Menacho Herbas

Anita Abreu de Carvalho

Karolina de Souza Cardoso

Cristhiano Chiovato Abdala 
DOI 10.22533/at.ed.39320031215

CAPÍTULO 16. 160

MICRORNAS DO REJUVENESCIMENTO: A ATUAÇÃO DA EPIGENÉTICA NA REGULAÇÃO FENOTÍPICA DO ENVELHECIMENTO CUTÂNEO

Rafael Carlos Bíscaro

Lilian Mussi

Jeanifer Caverzan da Silva

Bianca da Silva Sufi

Giovana Padovani

Lucas Idacir Sbrugnera Nazato

Flavio Bueno Camargo Junior

Wagner Vidal Magalhães

DOI 10.22533/at.ed.39320031216

CAPÍTULO 17

O RESGATE DO BRINCAR ATRAVÉS DA SEMANA MUNICIPAL DO BRINCAR: DA LEI A PRÁTICA

Débora Cristina Modesto Barbosa

Renata Miyake Almeida Prado

Pedro Martins Faria

Arieny Reche Silva

Alessandra Cristina Camargo Tarraf

Maria Clara Ferreira de Sousa Nóbrega

Leonardo Salamaia

Ana Gabriela Machado Nascimento

Camila da Fonseca e Souza Santos

Camila Arruda Dantas Soares

Ana Luiza Camilo Lopes

Beatriz Góes de Oliveira

DOI 10.22533/at.ed.39320031217

CAPÍTULO 18

PACIENTES SÉPTICOS - ESTUDO DOS CRITÉRIOS DE IDENTIFICAÇÃO NA EMERGÊNCIA DE UM HOSPITAL PÚBLICO DE BRASÍLIA

Taylla Rodrigues Chaves

Felipe Nogueira Affiune Silva

Priscilla Cartaxo Pierri Bouchardet

Noriberto Barbosa da Silva

Margô Gomes de Oliveira Karnikowski

Mauro Karnikowski

Leonardo Costa Pereira

Fabiana Xavier Cartaxo Salgado

DOI 10.22533/at.ed.39320031218

CAPÍTULO 19

PERFIL CLÍNICO-EPIDEMIOLÓGICO DOS PACIENTES NOTIFICADOS COM 
HANSENÍASE NO ESTADO DO MARANHÃO DE 2006 A 2015

Clístenes Alyson de Souza Mendonça

Christopher Andersennn de Souza Mendonça

Maria de Fátima Lires Paiva

Regina Maria Abreu Mota

Luana Karonine Cordeiro Castro

Rita da Graça Carvalhal Frazão Corrêa

Francisca Jade Lima de Andrade Silva

Diego Raí de Azevedo Costa

Dorlene Maria Cardoso de Aquino

DOI 10.22533/at.ed.39320031219

CAPÍTULO 20. 206

PERFIL DA INCIDÊNCIA E A PREVALÊNCIA DE HIPERTENSOS NO NORTE EM COMPARAÇÃO COM A REGIÃO SUDESTE

João Vitor Smith Martins

DOI 10.22533/at.ed.39320031220

CAPÍTULO 21 208

PERFIL EPIDEMIOLÓGICO DA MORTALIDADE INFANTOJUVENIL POR CAUSAS EXTERNAS EM PALMAS - TO: ANÁLISE DE 2009 A 2018

Amanda Moreno Costa

Laíz Soares Silva

Rayssa Mayra Figueira de Alencar

Delcides Bernardes da Costa Neto

DOI 10.22533/at.ed.39320031221

CAPÍTULO 22

PRÁTICAS DE CUIDADOS À RECÉM-NASCIDOS EM UNIDADES DE CUIDADOS ESPECIAIS

Silvana dos Santos Zanotelli

Danieli Parisotto

Denise Antunes de Azambuja Zocche

Vanessa Aparecida Gasparin

Andreia Cristina Dall'Agnol

DOI 10.22533/at.ed.39320031222

CAPÍTULO 23 233

PRESCRIÇÃO DE BISFOSFONATOS PARA MULHERES NA PÓS-MENOPAUSA: INDICAÇỐES E CONFLITOS DE INTERESSE

Bárbara Lacerda de Oliveira Faria

Clarissa Raquel da Silva Gomes

Filipe Salvador Zinatelli Coelho

DOI 10.22533/at.ed.39320031223

CAPÍTULO 24 241

PROGRAMA DA PRESSÃO ARTERIAL DA BEIRA BAIXA (ESTUDO PPABB) - FASE 1

Patrícia Margarida dos Santos Carvalheiro Coelho 
Francisco José Barbas Rodrigues

Inês Arvana Cheira Mourinha Mira

Tiago Joaquim Rodrigues Bernardes

Ana Teresa Fonseca Gomes

Débora Raquel Fernandes da Silva

Carla Carvalho Simões

Mariana Sofia Venâncio Batista

Sandra Marlene Sousa Rodrigues

Iolanda Cristina Carvalho Martins

Renata Oliveira Fazenda

DOI 10.22533/at.ed.39320031224

CAPÍTULO 25.

262

QUALIDADE DE VIDA DE INDIVÍDUOS COM DOENÇA RENAL CRÔNICA EM TRATAMENTO HEMODIALÍTICO

Isabela Santana Macedo

Gabriela Santana Macêdo

Edildete Sene Pacheco

Agna Roberta Rodrigues de Sousa

Aclênia Maria Nascimento Ribeiro

Luzia Fernandes Dias

Alaine Maria da Costa

Jardilson Moreira Brilhante

Maria do Socorro Marques do Nascimento Filha

Francisca das Chagas de Jesus Soares Oliveira

Gislane de Sousa Rodrigues

Gualbitânia de Sousa Oliveira Barbosa

DOI 10.22533/at.ed.39320031225

CAPÍTULO 26 273

RELATO DE EXPERIÊNCIA: GRUPO DE GESTANTES UBS JOY BETTS

Vanda Veridiana Cezar Parode

Idiana Vieira Pedroso

Tiele Giovana Almeida Santana

Andrea Janaina Martins de Souza

Gisela Cataldi Flores

DOI 10.22533/at.ed.39320031226

CAPÍTULO 27.

REVISÃO SOBRE O USO DO PLASMA RICO EM PLAQUETAS (PRP) NO TRATAMENTO DE ÚLCERAS VENOSAS

Alcione Matos de Abreu

Beatriz Guitton R. B. de Oliveira

Marcelle Feitosa Lemos Malveira

Nathalia Caldas Santos

DOI 10.22533/at.ed.39320031227 
TRAJETÓRIA DO INDIVÍDUO AMPUTADO DO PÓS-CIRÚRGICO À REABILITAÇÃO

Rodrigo Luis Ferreira da Silva

Bruno Pereira Bandeira

Jorge Carlos Menezes Nascimento Junior

DOI 10.22533/at.ed.39320031228

CAPÍTULO 29

TRANSTORNO DE ANSIEDADE EM MULHERES: UMA VISÃO HOLÍSTICA

Izadora Cristina Freitas Menezes

DOI 10.22533/at.ed.39320031229

SOBRE O ORGANIZADOR 


\section{PROGRAMA DA PRESSÃO ARTERIAL DA BEIRA BAIXA (ESTUDO PPABB) - FASE 1}

Data de aceite: 01/12/2020

Data de submissão: 04/09/2020

Patrícia Margarida dos Santos Carvalheiro Coelho Instituto Politécnico de Castelo Branco, Escola Superior de Saúde Dr. Lopes Castelo Branco - Portugal https://orcid.org/0000-0002-9862-0691

Francisco José Barbas Rodrigues Instituto Politécnico de Castelo Branco, Escola Superior de Saúde Dr. Lopes Castelo Branco - Portugal https://orcid.org/0000-0001-8405-4249

Inês Arvana Cheira Mourinha Mira Instituto Politécnico de Castelo Branco, Escola Superior de Saúde Dr. Lopes Castelo Branco - Portugal

Tiago Joaquim Rodrigues Bernardes Instituto Politécnico de Castelo Branco, Escola Superior de Saúde Dr. Lopes Castelo Branco - Portugal

\section{Ana Teresa Fonseca Gomes} Instituto Politécnico de Castelo Branco, Escola Superior de Saúde Dr. Lopes Castelo Branco - Portugal

\section{Débora Raquel Fernandes da Silva} Instituto Politécnico de Castelo Branco, Escola Superior de Saúde Dr. Lopes

Castelo Branco - Portugal

Carla Carvalho Simões Instituto Politécnico de Castelo Branco, Escola Superior de Saúde Dr. Lopes Castelo Branco - Portugal
Mariana Sofia Venâncio Batista Instituto Politécnico de Castelo Branco, Escola Superior de Saúde Dr. Lopes Castelo Branco - Portugal

Sandra Marlene Sousa Rodrigues Instituto Politécnico de Castelo Branco, Escola Superior de Saúde Dr. Lopes Castelo Branco - Portugal

Iolanda Cristina Carvalho Martins Instituto Politécnico de Castelo Branco, Escola Superior de Saúde Dr. Lopes Castelo Branco - Portugal

Renata Oliveira Fazenda Instituto Politécnico de Castelo Branco, Escola Superior de Saúde Dr. Lopes Castelo Branco - Portugal

RESUMO: Introdução: A hipertensão arterial é um problema de saúde pública que tem ganho uma grande expressão em Portugal. Esta patologia constitui um importante fator de risco para o acidente vascular cerebral e para o enfarte agudo do miocárdio, contribuindo significativamente para o aumento da morbilidade e mortalidade associado às patologias cerebrocardiovasculares. Objetivo: Determinar a prevalência de hipertensão arterial na população adulta da Beira Baixa e sua relação com os fatores de risco cerebrocardiovasculares. Materiais e métodos: Estudo do tipo transversal, analítico e observacional. A recolha da amostra decorreu em todos os concelhos da região da Beira Baixa com o objetivo de identificar a prevalência de hipertensão arterial nessas 
populações. Os dados foram recolhidos entre os anos de 2010 e 2014, perfazendo um total da amostra de 11316 indivíduos, dos quais 55,6\% são do sexo feminino ( $n=6292)$ e 44,4\% do masculino ( $n=5024)$. Resultados: A prevalência de hipertensão arterial encontrada foi de $52,55 \%$, com maior prevalência no sexo masculino $(53,2 \%)$ em relação ao feminino $(52,0 \%)$. Obteve-se uma prevalência de hipertensão arterial medicada de $37,7 \%$, em que 50,34\% apresentaram valores de pressão arterial controlados. Ao analisar os dados percebeu-se que a prevalência de hipertensão arterial de "novo" foi de 25,0\%. Quando estudados os fatores de risco associados constatou-se que os mais prevalentes foram o sedentarismo $(75,9 \%)$, a presença de história familiar de hipertensão arterial $(54,1 \%)$ e a hipercolesterolémia $(41,0 \%)$. Conclusão: Os resultados mostram uma elevada prevalência de hipertensão arterial na Beira Baixa e ainda que quase metade dos indivíduos com diagnóstico de hipertensão arterial não têm os valores de pressão arterial controlados.

PALAVRAS-CHAVE: Hipertensão Arterial, Prevalência, Fatores de Risco, Doenças Cardiovasculares.

\section{STUDY PPABB - 1ST FASE}

ABSTRACT: Introduction: Arterial hypertension is a public health problem that has gained great expression in Portugal. This pathology represents an important risk factor for stroke and acute myocardial infarction, contributing significantly to the increase in morbidity and mortality associated with cerebrocardiovascular diseases. Objective: To determine the prevalence of arterial hypertension in the adult population of Beira Baixa and its relationship with cerebrocardiovascular risk factors. Materials and methods: Cross-sectional, analytical and observational study. Sample collection took place in all counties in the Beira Baixa region in order to identify the prevalence of arterial hypertension in these populations. The data were collected between the years 2010 and 2014, making a total sample of 11316 individuals, of which $55.6 \%$ are female $(n=6292)$ and $44.4 \%$ male $(n=5024)$. Main results: The prevalence of arterial hypertension found was $52.55 \%$, with a higher prevalence in males $(53.2 \%)$ compared to females $(52.0 \%)$. A prevalence of medicated arterial hypertension of $37.7 \%$ was obtained, in which $50.34 \%$ had controlled blood pressure values. When analyzing the data, it was noticed that the prevalence of "new" arterial hypertension was $25.0 \%$. When the associated risk factors were studied, it was noticed that the most prevalent were sedentary lifestyle $(75.9 \%)$, the presence of a family history of arterial hypertension $(54.1 \%)$ and hypercholesterolemia (41.0\%). Conclusion: The results show a high prevalence of arterial hypertension in Beira Baixa and even though almost half of the individuals diagnosed with arterial hypertension do not have their blood pressure values controlled.

KEYWORDS: Arterial Hypertension, Prevalence, Risk Factors, Cardiovascular Disease.

\section{1। INTRODUÇÃO}

A hipertensão arterial (HTA) é considerada um grave problema de saúde pública e é a principal causa de morbilidade e mortalidade dos países desenvolvidos, especialmente quando é desconhecida ou mal controlada (GOMES, COELHO e PEREIRA, 2012; SIMÕES, COELHO, PEREIRA e PEREIRA, 2011; SKOREK, SOUZA e FREITAS, 2013), sendo 
definida como uma patologia multifatorial sistémica que se consegue diagnosticar através da avaliação da pressão arterial (PA) (BRITO, PANTAROTTO e COSTA, 2011; FERMINO, SEABRA, GARGANTA e MAIA, 2009).

Segundo Polónia et al, para existir um correto diagnóstico de HTA deve proceder-se à avaliação da PA pelo menos três vezes com um intervalo de tempo entre cada avaliação, assumindo-se o valor da PA o resultado do cálculo da média das avaliações (POLÓNIA, RAMALHINHO, MARTINS e SAAVEDRA, 2006). Segundo as orientações da Sociedade Europeia considera-se um indivíduo hipertenso quando atinge valores de PA sistólica (PAS) $\geq 140 \mathrm{mmHg}$ e/ou valores de PA diastólica (PAD) $\geq 90 \mathrm{mmHg}$ (SIMÕES, COELHO, PEREIRA e PEREIRA, 2011; MANCIA ET AL, 2013; WILLIAMNS, MANCIA, SPIERING, AGABITI ROSEI, AZIZI, BURNIER ET AL, 2018).

De acordo com os resultados dos estudos PAP e VALSIM a prevalência de HTA em Portugal é de 42,1\% e 42,6\%, respetivamente (MACEDO, LIMA, SILVA, ALCANTARA, RAMALHINO e CARMONA, 2007; CORTEZ-DIAS, MARTINS, BELO e FIUZA, 2009). Estes estudos demostram ainda que existe grande assimetria entre as diferentes regiões de Portugal, sendo o Alentejo o que apresenta a maior prevalência, com 49,5\% e a zona norte a menor prevalência, com 37,8\% (MACEDO, LIMA, SILVA, ALCANTARA, RAMALHINO e CARMONA, 2007). A região centro apresenta valores na ordem dos 45,4\% (GOMES, COELHO e PEREIRA, 2012), no entanto existe uma disparidade grande nesta região que é causada pelo litoral, mais desenvolvido e com hábitos de vida bastante diferentes do interior, onde está localizada uma grande taxa de indivíduos idosos e com hábitos alimentares típicos da região. Para além da HTA existem outros fatores de risco que podem influenciar os valores. De entre os mais comummente associados, destacam-se o sedentarismo, a obesidade, o alcoolismo, o tabagismo, a idade, o género, a hipercolesterolémia e a Diabetes Mellitus (DM) (GOMES, COELHO e PEREIRA, 2012; RIBEIRO e FARIA, 2013; CORDINHÃ, PAÚL E FERNANDES, 2009). No estudo AMALIA, estes fatores de risco apresentaram-se com prevalências elevadas, sendo os mais relevantes o sedentarismo (76,0\%), a obesidade $(51,6 \%)$ e a HTA $(23,5 \%)$ (PERDIGÃO, ROCHA, DUARTE, SANTOS e MACEDO, 2011)

A HTA apresenta-se como o fator de risco mais importante para as doenças cerebrocardiovasculares, nomeadamente o enfarte agudo do miocárdio (EAM) e o acidente vascular cerebral (AVC), que por sua vez são importantes causas de morbimortalidade em todo o mundo (CORDINHÃ, PAÚL E FERNANDES, 2009; PERDIGÃO, ROCHA, DUARTE, SANTOS e MACEDO, 2011). Em Portugal morrem cerca de 40 mil indivíduos/ano devido a estas duas patologias, em que cerca de 20 mil óbitos/ano ocorrem por AVC e 10 mil óbitos/ ano por EAM (MACEDO, LIMA, SILVA, ALCANTARA, RAMALHINO e CARMONA, 2007).

O Programa da Pressão Arterial surge da necessidade de perceber qual a prevalência de hipertensão arterial numa região do interior de Portugal - a Beira Baixa e de junto da população possibilitar uma adequada sensibilização e acompanhamento para 
as orientações mais recentes de HTA. Em conjunto com as autoridades de saúde locais pretende-se adotar medidas com o objetivo de reduzir as taxas de prevalência de HTA e das doenças cardiovasculares que a ela estão associadas na região estudada.

\section{I MATERIAIS E MÉTODOS}

Estudo transversal, analítico, observacional com recolha de dados prospetiva, sendo a amostra recolhida representativa de todos os concelhos da Beira Baixa, com recolha presencial pela equipa de investigadores (Belmonte, Covilhã, Sertã, Fundão, Proença-a-Nova, Vila de Rei, Idanha-a-Nova, Vila Velha de Rodão, Castelo Branco, Oleiros e Penamacor), entre os anos de 2010 e 2014.

Para o cálculo da amostra da população de cada concelho foram assumidos os dados dos CENSUS de 2011 (INE, 2011) publicados no site do Instituto Nacional de Estatística. Na seleção da amostra, e de acordo com critérios de inclusão definidos, foram considerados os indivíduos com idade superior ou igual a 18 anos, residentes nos diferentes concelhos, disponíveis para participar voluntariamente no estudo; como critério de exclusão foi definido, desde o início do estudo, que a recolha não poderia ser efetuada em locais como instituições de saúde ou lares de idosos de forma a não haver influência nos resultados encontrados.

\subsection{Protocolo do Estudo}

Para a recolha de dados foi necessário um esfigmomanómetro e braçadeira manuais (MDF instruments ${ }^{\circledR}$ ), uma balança digital $\left(\right.$ Sanitas ${ }^{\circledR}$ ), um questionário e o respetivo consentimento informado. Com o uso do questionário pretendíamos informações antropométricas (idade, peso e altura), informações sobre a avaliação da PA, terapêutica, atividade física, patologias cardíacas e a presença ou não de fatores de risco como hábitos tabágicos, colesterol e diabetes.

Em todos os locais de recolha da amostra foi distribuído um folheto com a descrição do estudo. Os indivíduos eram abordados aleatoriamente e convidados a participar, se aceitassem, era explicado todo o procedimento sendo obtido o peso, a frequência cardíaca e a avaliação manual da PA que foi efetuada com o individuo sentado, em repouso e avaliado por três vezes com um intervalo de tempo de cinco minutos entre cada uma.

Para o estudo do IMC, os indivíduos foram agrupados com base na classificação da OMS: tendo baixo peso se $<18,5 \mathrm{~kg} / \mathrm{m}^{2}$, peso normal se $18,5-24,9 \mathrm{~kg} / \mathrm{m}^{2}$, excesso de peso se $25-29.9 \mathrm{~kg} / \mathrm{m}^{2}$ e obesidade quando> $30 \mathrm{~kg} / \mathrm{m}^{2}$ (OMS). Foram estudados os hábitos tabágicos, a atividade física (englobaram-se todas as possíveis atividades), os antecedentes pessoais de doenças cardiovasculares (considerando-se como outras situações clínicas, a nível da exposição dos resultados, a miopericardite, miocardiopatia dilatada e sopro cardíaco), DM e dislipidémia. Foram ainda questionados os antecedentes familiares de HTA, tendo esta variável em consideração a presença da patologia em familiares diretos. 
Foi considerada HTA medida quando os valores médios resultantes das três avaliações de PA sistólica e PA diastólica, se encontravam acima dos limites da normalidade; a HTA medicada definida para todos os indivíduos que se encontravam a fazer medicação anti-hipertensiva; a HTA controlada para todos os indivíduos que se encontravam a fazer terapêutica anti-hipertensiva e que apresentaram valores de PA dentro da normalidade e, por fim, foi estipulada a prevalência de HTA geral para todos os indivíduos que apresentavam níveis elevados de PA e para todos os que se encontravam a fazer medicação antihipertensiva e que apresentavam valores de PA dentro da normalidade.

Quanto à classificação dos valores de PA, foram assumidos com base nas Guidelines de2013 da European Society of Hypertension e da European Society of Cardiology (MACIA, FAGARD, NARKIEWICZ, REDON, ZANCHETTI, BOHN, ET AL, 2013), que são corroborados pelas Guidelines de 2018 (WILLIAMNS, MANCIA, SPIERING, AGABITI ROSEI, AZIZI, BURNIER ET AL, 2018) considerando-se HTA de grau 1 (HTA ligeira) quando valores de PAS compreendidos entre 140-159 mmHg, e de PAD de 90-99 mmHg; HTA de grau 2 (HTA moderada) com valores de PAS entre 160-179 mmHg e de PAD entre 100-109 mmHg; e HTA de grau 3 (HTA grave) perante valores de PAS superiores ou iguais a 180 $\mathrm{mmHg}$ e de PAD superiores ou iguais a $110 \mathrm{mmHg}$. Para além destes graus foram ainda discriminados valores ótimos de PA ( $<120 \mathrm{mmHg}$ de PAS e $<80 \mathrm{mmHg}$ de PAD), valores de normalidade (PAS entre 120-129 mmHg e PAD entre $80-84 \mathrm{mmHg}$ ) e valores "borderline" (PAS entre 130-139 $\mathrm{mmHg}$ e PAD entre 85-89 $\mathrm{mmHg}$ ).

\subsection{Análise Estatística}

Os dados recolhidos foram inseridos, analisados e tratados com o recurso ao programa de análise estatística SPSS ${ }^{\circledR}$ (Statistical Product and Service Solution) versão 22.0. Recorreu-se a uma análise descritiva simples para caracterização da amostra e distribuição das variáveis, tendo-se calculado a frequência absoluta (n) e relativa (\%) para as variáveis qualitativas. Quanto às variáveis quantitativas, foram calculados a média, desvio padrão e valores mínimos e máximos. Foram estabelecidos como critério de significância estatística um $\mathrm{p} \leq 0,05$ e intervalo de confiança de 95\%.

De modo a averiguar as relações existentes entre diferentes variáveis, foram utilizados o teste qui-quadrado e o teste t-Student com a finalidade de analisar a diferença entre dois grupos de amostras independentes. Por fim, recorremos aos testes da correlação de Pearson para relacionar variáveis contínuas.

\section{3 Ética}

Todos os indivíduos foram informados dos procedimentos tendo sido obtido o consentimento informado, sob a forma escrita, ou verbal (no caso dos analfabetos). Os dados foram tratados de forma anónima, tendo sido todos classificados por código de números de forma a garantir a confidencialidade de todos os dados. O PPABB está 
aprovado por uma Comissão de Ética. A equipa de investigação declara não existirem conflitos de interesse na realização do presente estudo, tendo-se comprometido a respeitar os princípios expressos na declaração de Helsínquia.

\subsection{Amostra}

Do total da população da Beira Baixa obteve-se uma amostra representativa da população de 11316 indivíduos, em que 55,6\% eram do sexo feminino ( $n=6292)$ e 44,4\% do masculino ( $n=5024$ ) (gráfico 1), com idades compreendidas entre os 18 e 101 anos, uma média de 58,12 e desvio padrão de 17,96 anos.

Na distribuição das idades em função do sexo, percebe-se pela análise do Gráfico 1 , que a classe etária mais representativa é a dos 65-74 anos. Conclui-se também que existiu uma maior participação do género feminino em todas as classes etárias.

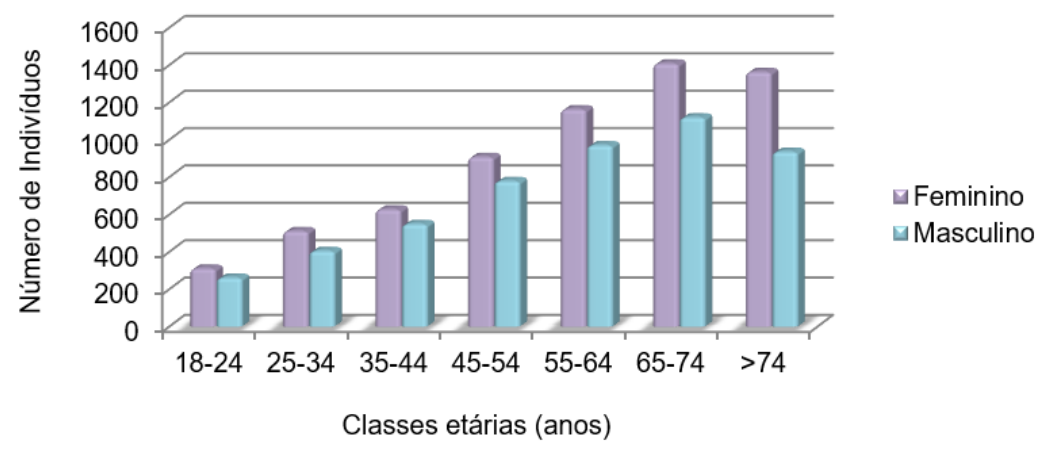

Gráfico 1 - Relação entre género e grupo etário $(n=11316)$

\section{I RESULTADOS}

\subsection{Perfil Antropométrico}

Da análise do IMC percebeu-se que a média deste índice foi de $26,62 \pm 4,11 \mathrm{Kg} / \mathrm{m}^{2}$, em que se obteve um valor mínimo de $15,57 \mathrm{Kg} / \mathrm{m}^{2}$ e máximo de $50,78 \mathrm{Kg} / \mathrm{m}^{2}$ o que revela que a maioria da amostra tem excesso de peso ou obesidade tal como se pode verificar no gráfico 2 que demonstra que quase metade da amostra $(44,7 \%)$ tem excesso de peso e $18,8 \%$ tem obesidade. Apenas $35,6 \%$ se encontra com o peso dentro dos valores de normalidade e $0,9 \%$ com baixo peso. 


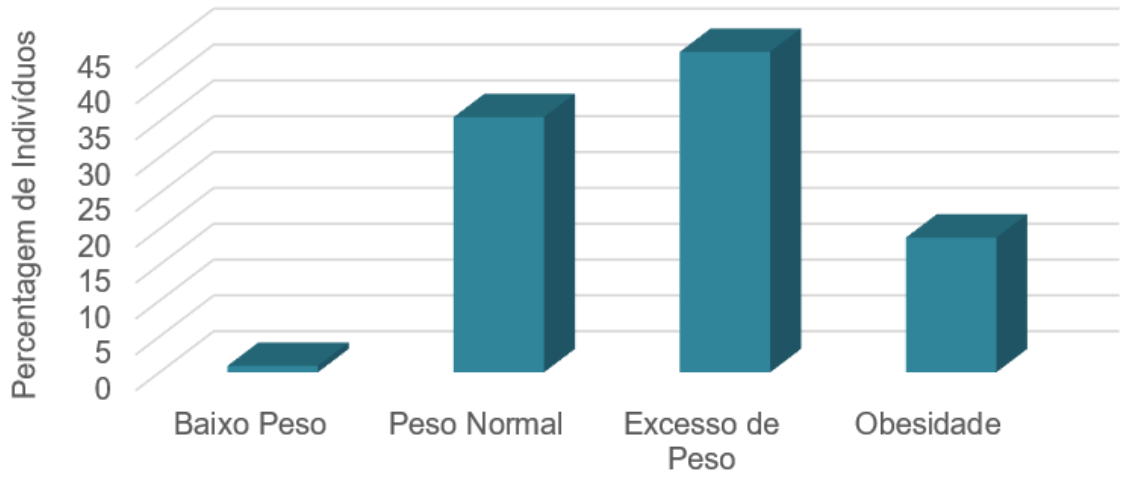

Gráfico 2 - Distribuição dos inquiridos por classes de Índice de Massa Corporal $(n=11310)$

\subsection{Fatores de Risco Cerebrocardiovasculares}

Quanto aos fatores de risco, tal como se pode observar no gráfico 3, os mais prevalentes foram o sedentarismo, a história familiar de HTA e a hipercolesterolémia, com $75,9 \%, 54,1 \%$ e $41,0 \%$, respetivamente.

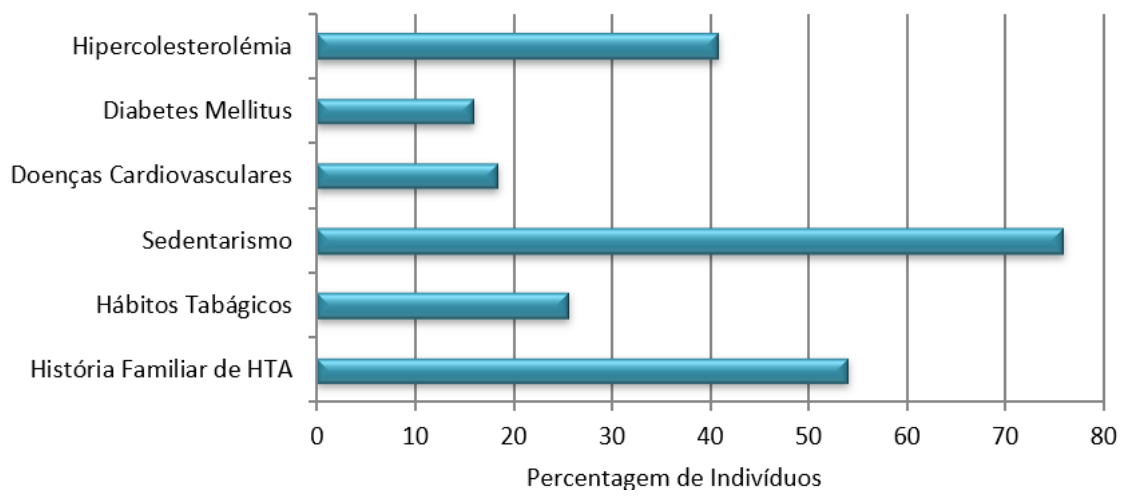

Gráfico 3 - Fatores de risco presentes na amostra $(n=11316)$

Verificou-se ainda, no decorrer desta análise, que 25,7\% dos inquiridos tinham hábitos tabágicos e 16,0\% apresentava DM. Desta análise importa referir que 18,4\% dos inquiridos assinalou ter presença de doenças cérebro e cardiovasculares. Desta forma, foi importante perceber que tipo de patologia poderiam ter diagnosticada: verificámos que, do total dos $18,4 \%$ a maioria respondeu ter a presença de Síndrome Coronorária Aguda (38,5\%); $28,4 \%$ perturbações de ritmo; $14,6 \%$ AVC e $8,6 \%$ presença de valvulopatia tal como se pode analisar no gráfico 4 . 


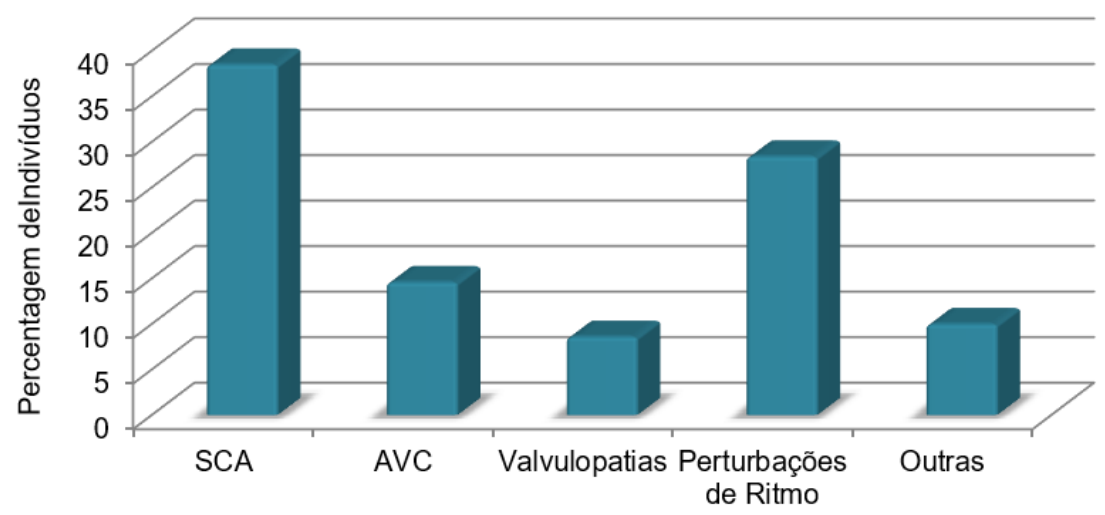

Gráfico 4 - Patologia cérebro e cardiovascular conhecida na amostra $(n=1167)$ Legenda: SCA- Síndrome Coronária Aguda; AVC - Acidente Vascular Cerebral

\subsection{Análise da Pressão Arterial Média}

No estudo da PA, verificámos que os valores médios da PAD foram de 77,48 $\pm 11,65$ mmHg com um valor mínimo de $40 \mathrm{mmHg}$ e máximo de 141,67 mmHg.

No gráfico 5 podemos observar a relação da PAD média com os sexos e por grupo etário, esta análise mostra-nos que os valores de PAD média foram superiores no sexo masculino em todas as classes etárias e que esta tende a aumentar à medida que a idade avança; o efeito da idade, porém, não se manifesta de maneira sequencial, dado que em ambos os géneros se verificou uma diminuição a partir das classes dos 55-64 anos.

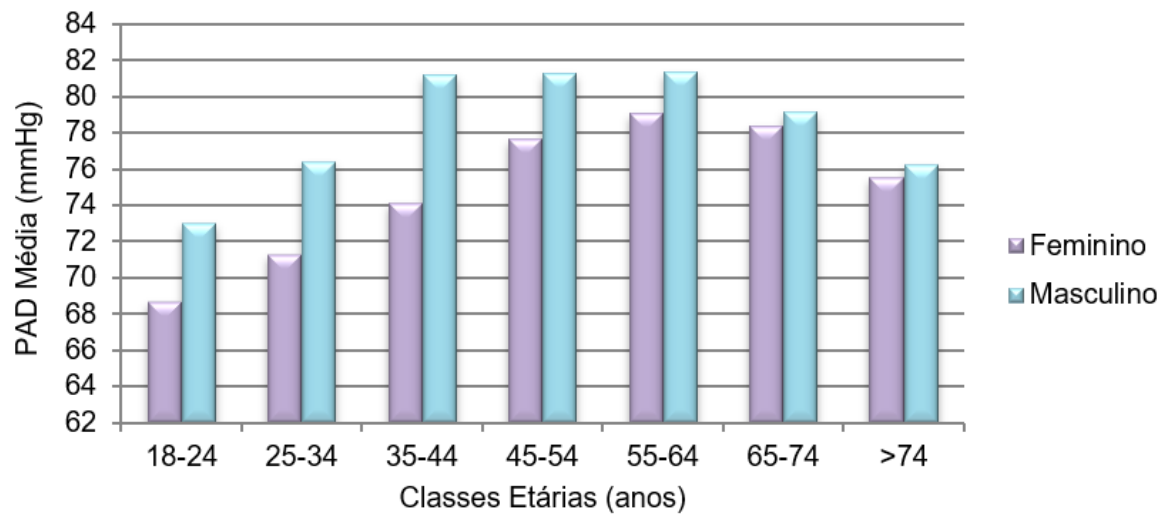

Gráfico 5 - Relação da PAD média com ambos os sexos e grupos etários ( $n=11313)$ 
Recorremos ao teste do t-Student e verificámos que existe alta significância estatística entre a PAD média e a idade $(p<0,001)$ e o género $(p<0,001)$.

Analisámos de seguida os valores de PAS, tendo sido obtido uma média de 128,66 $\pm 19,20 \mathrm{mmHg}$ com um valor mínimo de $76,67 \mathrm{mmHg}$ e máximo $230,00 \mathrm{mmHg}$.

No gráfico 6 apresenta-se a relação da PAS média com o sexo e o grupo etário, verificando-se assim que é no sexo masculino que se obtém os valores de PAS mais elevados até aos 74 anos.

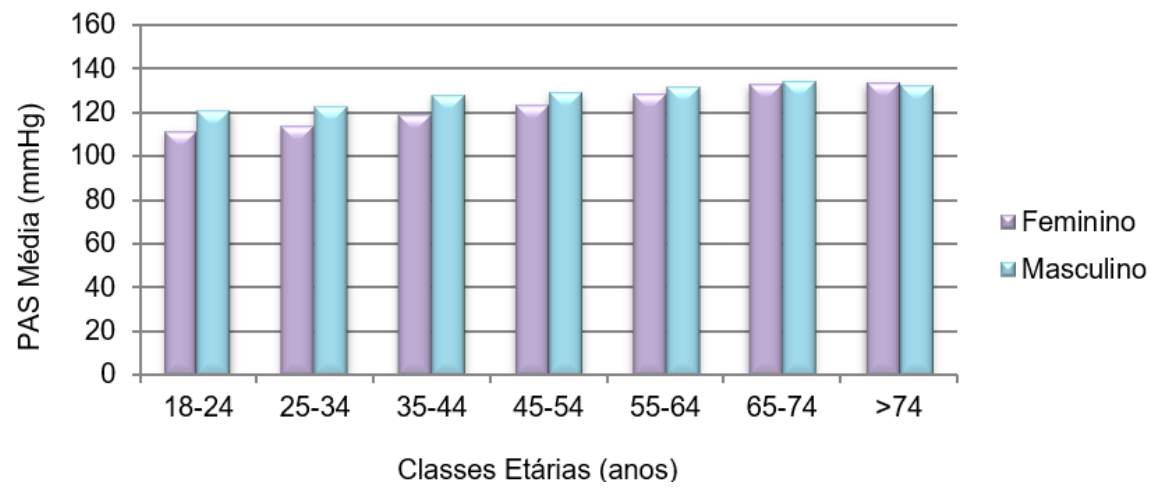

Gráfico 6 - Relação da PAS média com ambos os sexos e grupos etários $(n=11313)$

À semelhança do que se fez com a PAD recorreu-se ao teste do t-Student e verificouse que existe alta significância estatística entre a PAS e a idade $(p<0,001)$ e entre PAS e o $\operatorname{sexo}(p<0,001)$.

\subsection{Prevalência de Hipertensão Arterial}

Baixa

\subsubsection{Prevalência de Hipertensão Arterial na população adulta na Beira}

A prevalência de HTA encontrada no distrito de Castelo Branco, zona geográfica denominada como Beira Baixa, foi de $52,55 \%$, sendo que o sexo masculino $(53,2 \%)$ apresentou valores superiores em relação ao feminino (52,0\%).

Relacionou-se a prevalência de HTA com ambos os géneros e a faixa etária, tal como se pode analisar no gráfico 7 é possível perceber que à medida que a idade aumenta, vai aumentando também a prevalência de HTA em ambos os géneros. Pela análise gráfica concluiu-se ainda que a maior prevalência de HTA se encontra na classe etária dos indivíduos com idade superior a 74 anos. 


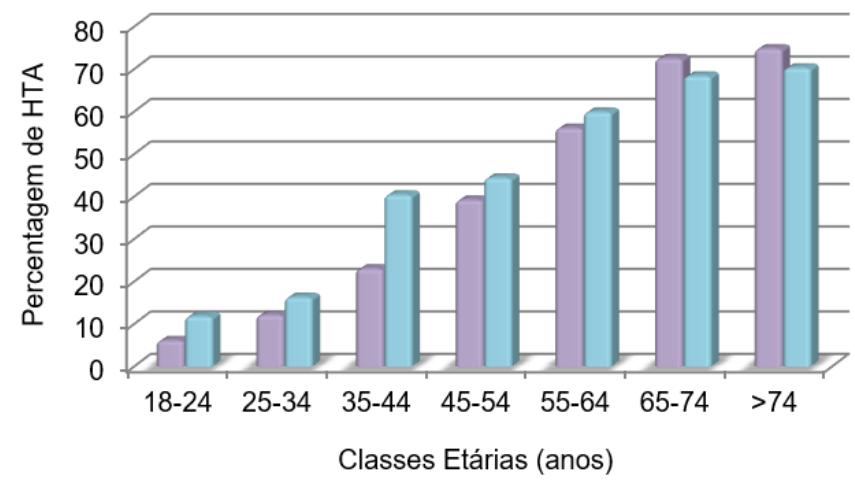

$\square$ Feminino $\square$ Masculino

Gráfico 7 - Relação da prevalência de HTA com ambos os sexos e grupos etários ( $n=11313)$

Foi estimada a percentagem de HTA por cada concelho da região da Beira Baixa, tal como se pode observar no gráfico 8 o concelho da Covilhã $(70,3 \%)$ é o que apresenta uma prevalência maior, seguido do concelho de Vila Velha de Rodão $(62,5 \%)$ e do de Oleiros $(56,7 \%)$, por outro lado os concelhos de Penamacor $(45,2 \%)$, Castelo Branco $(46,1 \%)$ e Belmonte $(46,6 \%)$ são os que apresentam menores prevalências de HTA.

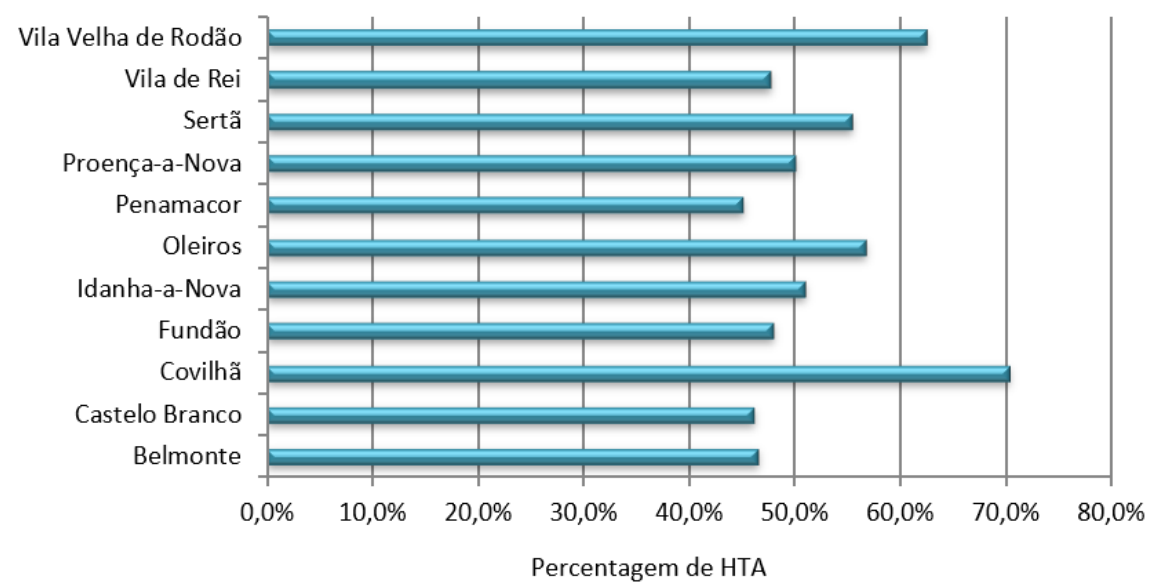

Gráfico 8 - Distribuição de HTA pelos concelhos da região da Beira Baixa $(n=11316)$

\subsubsection{Relação existente entre HTA e os diferentes fatores de risco}

Com o objetivo de se perceber se existia relação entre a HTA e as variáveis género e a idade, utilizou-se o teste do qui-quadrado e concluiu-se não existir qualquer relação com o género $(p=0,223)$, no entanto a hipertensão arterial está associada à idade com uma alta significância estatística entre ambas $p<0,001$. 
De seguida relacionámos a HTA geral com o IMC e percebemos que a prevalência de HTA geral é superior em indivíduos com excesso de peso. Para poder confirmar esta análise recorremos aos testes do qui-quadrado e t-Student, tendo-se verificado que existe uma alta significância estatística entre ambas as variáveis com um $p<0,001$, o mesmo se verificou para a relação com o sedentarismo, as doenças cardiovasculares, a DM, a hipercolesterolémia e a história familiar de HTA $(p<0,001)$.

\subsubsection{Prevalência de Hipertensão Arterial Medida}

No que diz respeito à HTA medida os valores encontrados mostram que $33,81 \%$ da população tem valores aumentados da Pressão Arterial, nesta variável estão incluídos os indivíduos que sabem ter HTA e que não está controlada e os indivíduos que desconhecem ter hipertensão.

Da análise do gráfico 9, onde se relaciona a prevalência de HTA medida com os sexos e os grupos etários, verificamos que o sexo masculino obteve valores superiores em todas as classes etárias, à exceção do grupo com idades entre os 65-74 anos em que o género feminino é ligeiramente mais prevalente, sendo também o grupo onde se verifica a maior percentagem de HTA (43\%). Conseguimos ainda perceber que, à medida que a idade aumenta, a HTA medida também aumenta até à classe etária dos 65-74 anos, o que não se verificou para as idades superiores a 74 anos, em que houve uma ligeira diminuição em relação ao grupo anterior.

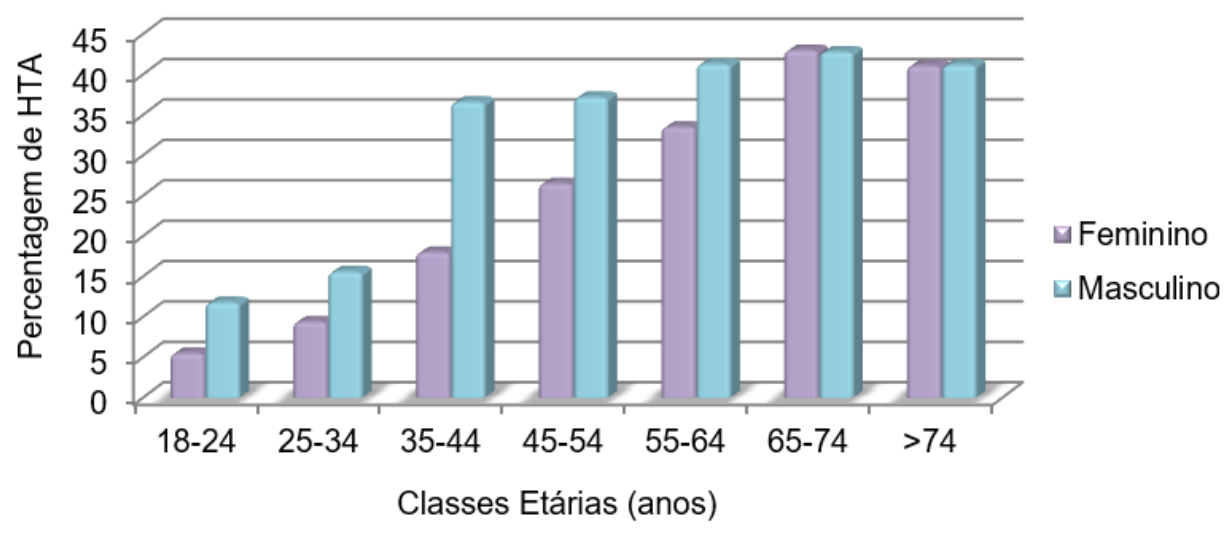

Gráfico 9 - Relação da prevalência de HTA medida em ambos os sexos com grupos etários $(n=11313)$

Foi estimada a percentagem de HTA medida em cada concelho estudado, podendo verificar-se, pelo gráfico 10, que os concelhos da Covilhã $(49,3 \%)$, Sertã $(38,8 \%)$ e Castelo Branco $(38,3 \%)$ são os que apresentam as maiores prevalências de HTA medida, ao 
contrário de Belmonte (23,4\%), Fundão (23,8\%) e Proença-a-Nova $(25,4 \%)$ que apresentam as menores percentagens.

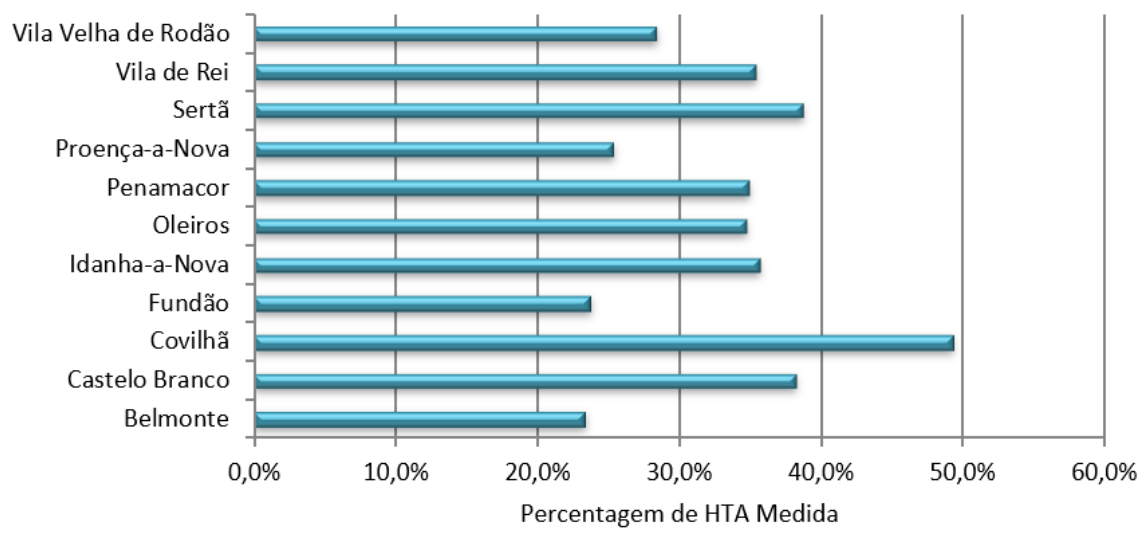

Gráfico 10 - Distribuição de HTA medida pelos concelhos estudados ( $n=11316)$

\subsubsection{Prevalência de Hipertensão Arterial Medicada}

A prevalência de HTA medicada foi de $37,72 \%$, sendo o sexo feminino $(41,0 \%)$ o grupo que apresentou uma maior percentagem quando comparado com o masculino (33,6\%). Quando a relacionámos com o sexo e as classes etárias percebemos que o sexo feminino é mais prevalente em todas as classes etárias e que à medida que a idade aumenta esta prevalência aumenta progressivamente também em todas as classes etárias. Concluiu-se ainda que a maior prevalência de HTA medicada se situa na classe etária $>74$ anos no género feminino $(65,6 \%)$.

\subsubsection{Prevalência de Hipertensão Arterial Controlada e Não Controlada}

De acordo com o analisado o valor de HTA medicada foi de $37,72 \%$, destes, metade apresentou os valores de PA dentro da normalidade, isto é, 50,34\% tinha HTA controlada e 49,66\% tinham os valores de PA não controlados, tal como se pode observar no gráfico 11 . 


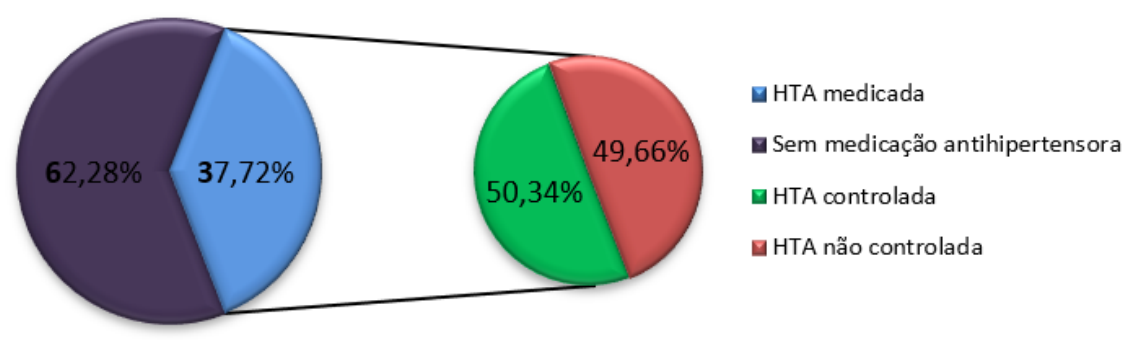

Gráfico 11 - Prevalência de HTA medicada, HTA não controlada, controlada e percentagem de indivíduos que não tem terapêutica anti-hipertensora

Ao realizar o cruzamento da HTA medicada com os sexos, verificámos que o feminino tem um valor de HTA controlada superior $(51,0 \%)$ comparado com o masculino, que apresentou uma percentagem de 49,3\%. Fizemos o mesmo exercício para os grupos etários e percebemos que esta é mais predominante no sexo feminino nos grupos etários 18-24, 25-34, 45-54 e >74 anos, e para o masculino nos grupos 35-44, 55-64 e 65-74 anos. Desta relação verificámos que a HTA controlada aumenta em todas as classes etárias à medida que a idade avança no sexo feminino, enquanto no masculino ela aumenta até aos 65-74 anos e depois sofre uma diminuição a partir dos 74 anos. Isto pode dever-se ao facto de termos mais indivíduos do género feminino no último grupo etário.

No exercício do estudo da prevalência de HTA não controlada percebemos que é mais predominante no sexo feminino nas classes etárias dos 18-24, 25-34, 45-54 e >74 anos. Observou-se ainda que a evolução da prevalência da HTA não controlada ao longo da idade, no género feminino, aumenta em todas as classes etárias, enquanto no masculino aumenta até aos 65-74 anos, onde se verifica a maior percentagem de HTA não controlada (35\%), e depois sofre uma diminuição no último grupo etário (Gráfico 12). 


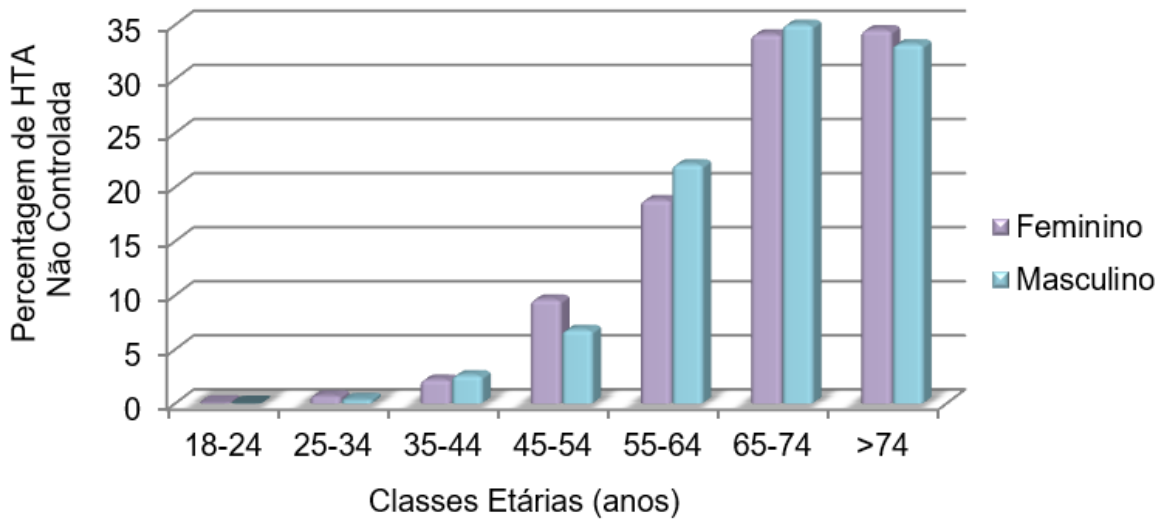

Gráfico 12 - Relação da prevalência de HTA não controlada com o sexo e grupos etários $(\mathrm{N}=4210)$

Estudámos as percentagens da HTA não controlada em cada um dos concelhos da região estudada, gráfico 13 e contatámos que os concelhos com maior prevalência de HTA não controlada foram os concelhos de Castelo Branco (77,2\%), Covilhã $(60,9 \%)$ e Penamacor $(57,0 \%)$, enquanto os concelhos do Fundão $(31,1 \%)$, Belmonte $(32,4 \%)$ e Vila Velha de Rodão $(37,2 \%)$ apresentam valores mais baixos de HTA não controlada.

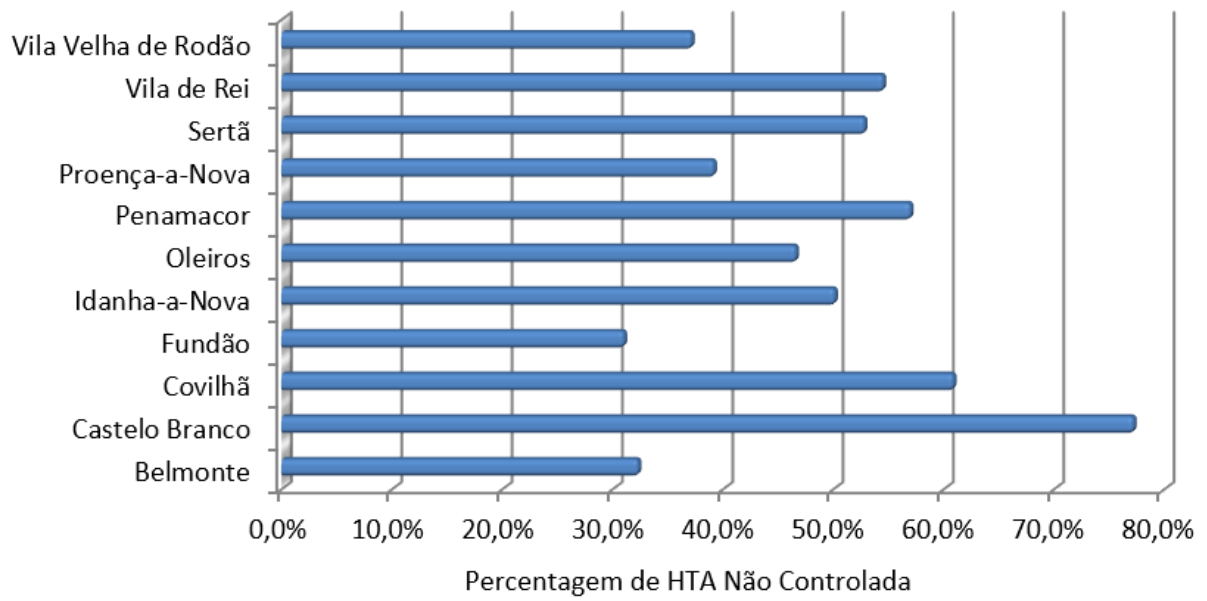

Gráfico 13 - Distribuição de HTA não controlada pelos concelhos da Beira Baixa ( $\mathrm{N}=4211)$

\subsubsection{Prevalência de Hipertensão Arterial de "Novo"}

Por fim, estudámos a prevalência da HTA de "novo", que se refere aos casos em que a avaliação da PA revelou valores de hipertensão nos indivíduos que desconheciam ser 
hipertensos, onde se obteve uma prevalência de $24,95 \%$, sendo $30,5 \%$ no sexo masculino e 19,9\% feminino. Ao relacionar esta variável com o sexo e os grupos etárias, verificou-se que o género masculino é o mais prevalente em todas as grupos etários, constatando-se para ambos os sexos ainda que à medida que a idade aumenta, a prevalência de HTA de novo aumenta em todas as classes etárias conforme se pode observar no gráfico 14.

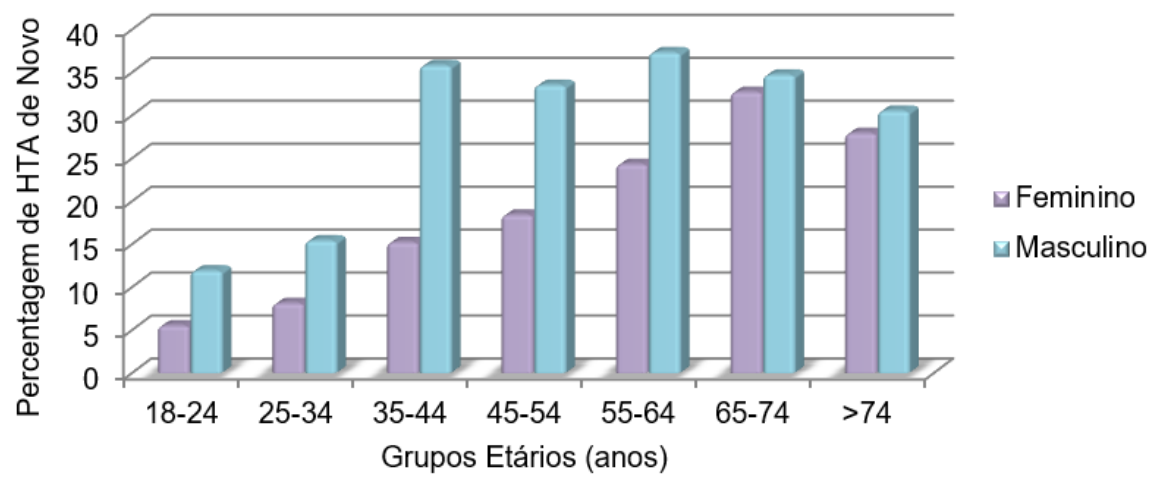

Gráfico 14 - Relação da prevalência de HTA de "novo" em ambos sexos e grupos etários $(n=6951)$

Estudámos ainda as percentagens da HTA de "novo" em cada um dos concelhos e constatámos, tal como se pode observar no gráfico 15, que os concelhos com maior prevalência de HTA de "novo" são os da Covilhã (35,8\%), Sertã $(32,1 \%)$ e Idanha-a-Nova $(29,3 \%)$, enquanto os concelhos de Proença-a-Nova (16\%), Vila Velha de Rodão $(18,1 \%)$ e Belmonte $(18,7 \%)$ apresentam as menores percentagens.

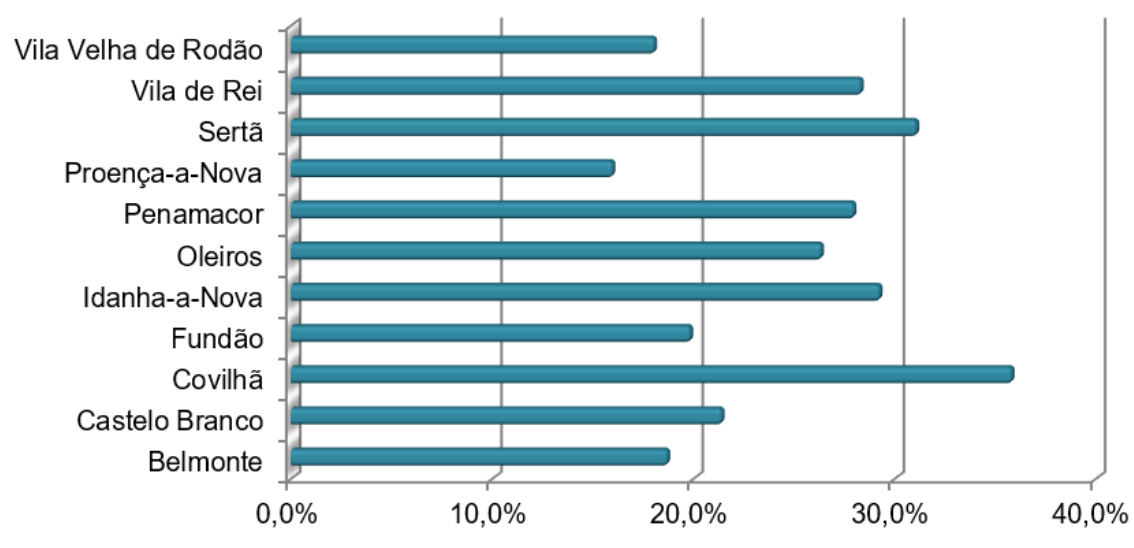

Percentagem de HTA de Novo

Gráfico 15 - Distribuição de HTA de "novo" pelos concelhos da Beira Baixa $(n=6953)$ 


\section{I DISCUSSÃO E CONCLUSÕES}

Apesar da evolução nos últimos anos ao nível do conhecimento, tratamento e controlo da HTA, esta patologia continua a ser um grave e crescente problema de saúde pública pela mortalidade e morbilidade que Ihe está associada. Em consequência desta problemática, têm surgido vários projetos, com objetivos de promoção e educação para o controlo da HTA. O PPABB vem neste encontro, tendo como principal objetivo a contribuição para o conhecimento da prevalência desta patologia na região da Beira Baixa.

A HTA é hoje uma patologia que é considerada um grave problema de saúde pública, apresenta valores de prevalência bastante elevados (GOMES, COELHO e PEREIRA, 2012; SIMÕES, COELHO, PEREIRA e PEREIRA, 2011). A nível nacional, a prevalência de HTA ronda os 42,1\% (FERMINO, SEABRA, GARGANTA e MAIA, 2009), valor muito próximo ao da Europa que é de 40,0\%. Já nos EUA a prevalência é apontada nos 28,0\% (CORTEZDIAS, MARTINS, BELO e FIUZA, 2009).

O PPABB estudou, numa primeira fase, a prevalência de HTA em todos os concelhos da Beira Baixa - distrito de Castelo Branco que se situa no interior centro de Portugal. Verificou-se que mais de metade da população tem valores de PA aumentados, com uma prevalência de HTA de 52,55\%, valor acima dos encontrados nos estudos PAP, VALSIM, AMALIA e Rocha et al, que revelam prevalências de HTA de 42,1\%, 42,62\%, 23,5\% e 44\%, respetivamente (MACEDO, LIMA, SILVA, ALCANTARA, RAMALHINHO E CARMONA, 2007; CORTEZ-DIAS, MARTINS, BELO e FIUZA, 2009; PERDIGÃO, ROCHA, DUARTE SANTOS e MACEDO, 2011; ROCHA, MELLO E SILVA e NOGUEIRA, 2003).

Relativamente à distribuição da percentagem de HTA por sexo, percebe-se que é o sexo masculino que apresenta uma percentagem de HTA superior, 53,2\%, comparativamente ao feminino, 52,0\%. No entanto, através do teste do qui-quadrado, verificámos que não existe uma relação estatisticamente significativa entre estas variáveis $(p=0,223)$. Ao comparar os resultados encontrados pela equipa de investigadores do PPABB com outros estudos, percebe-se que os valores encontrados são ligeiramente superiores aos dos de Pereira et al, em que a prevalência de HTA no género feminino foi de $42,7 \%$ e no masculino de $46,7 \%$ (PEREIRA, AZEVEDO e BARROS, 2010) e no estudo de VALSIM que revela uma prevalência de $42,19 \%$ no sexo feminino e no masculino de 43,09\% (CORTEZ-DIAS, MARTINS, BELO e FIUZA, 2009).

Na relação efetuada entre a HTA e os grupos etários verificámos que a prevalência de HTA tem tendência a aumentar ao longo dos grupos etários, sendo que nas idades superiores a 74 anos se obtiveram as maiores percentagens, o que também vai ao encontro dos estudos VALSIM e PAP, em que se demonstra que existe uma evolução progressiva ao longo das classes etárias( CORTEZ-DIAS, MARTINS, BELO e FIUZA, 2009; MACEDO, LIMA, SILVA, ALCANTARA, RAMALHINHO E CARMONA, 2007). Realizou-se ainda o teste do qui-quadrado para se perceber se existia relação entre a idade e a presença de 
HTA, tendo-se aferido alta significância estatística entre ambas as variáveis $(p<0,001)$, podendo afirmar-se que a idade é um fator de risco para o desenvolvimento da HTA. De acordo com o estudo Gonzaga et al, a idade promove o desenvolvimento da HTA devido a dois acontecimentos, primeiro existe um aumento na produção de colagénio pelas células musculares lisas das artérias e, seguidamente, pode-se observar na população idosa a existência do aumento da sensibilidade ao sódio e a deficiência de óxido nítrico, que por consequência aumenta o stress oxidativo. As combinações destas alterações favorecem a vasoconstrição e diminuem a complacência arterial interferindo na distensibilidade dos vasos (GONZAGA, SOUZA e AMODEU, 2009).

Os indivíduos obesos apresentam alterações hemodinâmicas que se podem caraterizar pelo aumento da atividade do sistema simpático, pela redução da atividade da renina plasmática, pela expansão do volume plasmático, pelo débito cardíaco elevado e pela diminuição da resistência vascular periférica (ROSA, FARIA e LERÁRIO, 2000; JORDAN, YUMUK e SCHALAICH, 2012). Uma das consequências que está diretamente relacionada com a presença de HTA e a obesidade é o desenvolvimento da hipertrofia ventricular. Esta desenvolve-se devido ao aumento que existe das necessidades metabólicas por parte do excesso de tecido adiposo que por sua vez vai aumentar o trabalho cardiovascular (ROSA, FARIA e LERÁRIO, 2000; JORDAN, YUMUK e SCHALAICH, 2012). Ao estudar o IMC verificámos $63,5 \%$ da amostra tinha excesso de peso e obesidade, o que são valores extremamente alarmantes e que estão acima dos do estudo AMALIA onde é revelado uma percentagem de e excesso de peso de 51,6\% (PERDIGÃO, ROCHA, DUARTE, SANTOS e MACEDO, 2011). Também o estudo PAP apresenta uma elevada prevalência de excesso de peso e obesidade apresentando cerca de 50,0\% dos inquiridos com valores de IMC superiores à normalidade (MACEDO, LIMA, SILVA, ALCANTARA, RAMALHINHO e CARMONA, 2007) e no estudo de Silva, et al (estudo sobre a população de Florianápolis) que mostra que a prevalência de excesso de peso é de $51,9 \%$ e a de obesidade é $16,1 \%$, valores semelhantes aos por nós encontrados quando somamos as duas percentagens. Ao relacionar a HTA com o IMC concluímos que a percentagem onde se encontrava mais indivíduos com presença de HTA foi no grupo de excesso de peso, pelo teste do quiquadrado percebeu-se assim existir uma alta significância estatística entre a presença de HTA e o IMC $(p<0,001)$.

Foram ainda estudados as relações entre a HTA e o sedentarismo, a presença de história familiar de HTA e a hipercolesterolémia, com 75,9\%, 54,1\% e 41,0\%, respetivamente. Todos apresentam relação com a presença de HTA com um $p<0,001$, indo estes resultados também ao encontro dos resultados do estudo AMALIA, que apresenta como fator de risco mais prevalente o sedentarismo, 65,3\%. Ainda no estudo AMALIA (PERDIGÃO, ROCHA, DUARTA, SANTOS e MACEDO,2011) verificámos, na relação dos dados, que a hipercolesterolémia é muito menor $(19,7 \%)$ comparativamente com os resultados obtidos por nós. Já quanto ao sedentarismo os valores por nós encontrados 
foram muito elevados, encontrámos um outro estudo de Silva et al que também revela percentagens elevadas (53,1\%) (SILVA, PETROSKI e PERES, 2012), no entanto, inferior às por nós apresentadas. A alta prevalência de sedentarismo existente pode-se explicar pela crescente urbanização que levou a alterações nos comportamentos da sociedade, em que cerca de $57,0 \%$ da população europeia não pratica atividade física (MACHADO, ALVES, TINOCO, GONÇALVES, MATOS e REGO, 2010; KNOOPS, GROOT, KROMHOUT, PERRIN e MENOTTI, 2004; ARITA, MIYASHITA, MORIOKA e SHIRAISHI, 2002; MONTEIRO e SOBRAL, 2004; FORJAZ e TINUCCI, 2000; RUIVO e ALCANTARA, 2012). Contudo está comprovado que o exercício físico, quando bem implementado permite a diminuição da morbilidade e mortalidade cardiovascular (MACHADO, ALVES, TINOCO, GONÇALVES, MATOS e REGO, 2010; KNOOPS, GROOT, KROMHOUT, PERRIN e MENOTTI, 2004; ARITA, MIYASHITA, MORIOKA e SHIRAISHI, 2002; MONTEIRO e SOBRAL, 2004; FORJAZ e TINUCCI, 2000; RUIVO e ALCANTARA, 2012), tem uma influência positiva na dislipidémia, insulinorresistência, obesidade e na presença de HTA ou prevenção. Está descrito que uma prática de exercício físico regular pelo menos 3 vezes por semana por um período superior a 30 minutos trás benefícios para a saúde (MACHADO, ALVES, TINOCO, GONÇALVES, MATOS e REGO, 2010).

Analisámos ainda a prevalência dos hábitos tabágicos, das doenças cardiovasculares e da DM, que apresentaram, respetivamente, prevalências de 25,7\%, 18,4\% e 16,0\%. Em relação às doenças cardiovasculares: dos 18,4\% indivíduos que responderam ter uma destas patologias, 38,5\% referiu estar relacionado com a síndrome coronária aguda e $14,6 \%$ referiu antecedentes de AVC, o que indica que metade destes indivíduos tem patologia cérebro e cardiovascular. Para verificar a existência de uma relação estatística entre estes fatores e a HTA realizou-se o teste do qui-quadrado que revelou existir alta significância estatística $(p<0,001)$. Se compararmos os resultados com os do estudo AMALIA percebemos que quer em relação à $\mathrm{DM}(8,9 \%)$ quer ao tabagismo (16,3\%) este apresentou uma prevalência mais baixa (PERDIGÃO, ROCHA, DUARTE, SANTOS e MACEDO, 2011), já no estudo de Gus et al o tabagismo apresentou uma prevalência superior ao estudo PPABB, nomeadamente, 34,0\% (GUS, ZASLAVSKY, MEDINA e HARZHEIM, 2004).

Dos $37,72 \%$ de HTA medicada encontrada, cerca de metade da amostra demonstrou ter os valores da PA dentro da normalidade $(50,34 \%)$ valor muito superior ao encontrado no estudo $\operatorname{PAP}^{(8)}(11,2 \%)$. Estudámos ainda a prevalência de HTA de novo na Beira Baixa, onde obtivemos uma percentagem de $24,95 \%$, que se apresentou maior no género masculino $(30,5 \%)$ em relação ao feminino (19,9\%). Na mesma linha de pensamento, existe o estudo PAP, que mostra que 53,9\% não sabia que tinham HTA o que se demostrou bastante superior aos nossos resultados. Ainda no estudo PAP, conclui-se que a percentagem foi superior no género masculino $(63,3 \%)$ em relação ao feminino $(43,9 \%)$ (MACEDO, LIMA SILVA, ALCATARA, RAMALHINO e CARMONA, 2007) tal como por nós apresentado. 
Ao fazer uma análise da prevalência das várias formas de apresentação de HTA estudadas pelos concelhos da região concluímos que a Covilhã foi o concelho que apresentou prevalências de HTA (geral, medida, medicada, não controlada e de novo) mais elevadas. Em contrapartida, os concelhos que não estão tão perto de um centro hospitalar têm percentagens de HTA menos prevalentes, nomeadamente, Vila de Rei com HTA geral de 47,7\%, Belmonte com 23,4\% de HTA medida, Penamacor com 23,9\% de HTA medicada, Fundão com 31,1 de HTA não controlada e Proença-a-Nova de 16\% de HTA de novo.

Está descrito que a nível mundial cerca de $6,0 \%$ da população morre devido a existência de HTA não controlada (CORTEZ-DIAS, MARTINS, BELO e FIUZA, 2009), o que pode passar apenas pelo controlo dos fatores de risco modificáveis. Verificámos que a prevalência de HTA não controlada é demasiado elevada, uma vez que $49,66 \%$ dos indivíduos que realizam terapêutica anti-hipertensora têm os valores não controlados.

Visto que o nosso estudo apresentou resultados que confirmam a elevada prevalência de HTA nesta região do país, seja por mau controlo dos diversos fatores de risco ou pela falta de ajuste terapêutico, é necessário que se adotem medidas de prevenção primárias e secundárias para esta patologia.

Embora esteja garantida a representatividade da amostra do nosso estudo, esta nem sempre foi obtida aleatoriamente revelando-se por vezes ser uma recolha por conveniência na recolha dos diversos fatores de risco, que foram baseados na informação dada pelos inquiridos através da aplicação de questionário, o que nos levou a redesenhar alguns aspetos do estudo PPABB e permitiu avançar com um novo estudo (fase 2) com recolha de amostra aleatória por clusters e a introdução de novas variáveis que estão a ser implementadas para serem estudadas, tal como a prevalência de hipotensão ortostática que se tem revelado como um importante marcados de risco cerebrocardiovascular.

\section{REFERÊNCIAS}

GOMES AT, COELHO P, PEREIRA A. Prevalência de Hipertensão Arterial na População Adulta do Concelho da Covilhã. Revista Portuguesa de Hipertensão e Risco Cardiovascular, 2012;32:4-14.

SIMÕES C., COELHO P., PEREIRA A., PEREIRA T.. Prevalência de Hipertensão Arterial na Cidade de Castelo Branco. Revista Portuguesa de Hipertensão e Risco Cardiovascular, 2011;21:11-5.

SKOREK J., SOUZA R., FREITAS J,. Fatores de risco associados à hipertensão arterial sistêmica em comunidade da periferia de Anápolis. Estudos, 2013;40(2):165-75.

BRITO E., PANTAROTTO R., COSTA L. A hipertensão arterial sistêmica como fator de risco ao acidente vascularencefálico (AVE). Journal of the Health Sciences Institute, 2011;29(4):265-8.

FERMINO R., SEABRAA., GARGANTA R., MAIA J. Fatores Genéticos na Agregação Familiar da Pressão Arterial de Famílias Nucleares Portuguesas. Arquivos Brasileiros de Cardiologia, 2009;9(3):209-15. 
POLÓNIA J., RAMALHINHO V., MARTINS L., SAAVEDRA J. Normas Sobre Detecção, Avaliação e Tratamento da Hipertensão Arterial da Sociedade Portuguesa de Hipertensão. Revista Portuguesa de Cardiologia, 2006;25(6):649-60.

MANCIA G. et al. 2013 ESH/ESC Guidelines for the Management of Arterial Hypertension. Journal of Hypertension, 2013;31:1281-357.

WILLIAMNS B., MANCIA G., SPIERING W., AGABITI ROSEI E., AZIZI M., BURNIER M., et al. 2018 ESC/ESH Guidelines for the management of arterial hypertension: The Task Force for the management of arterial hypertension of the European Society of Cardiology (ESC) and the European Society of Hypertension (ESH). Eur Heart J, 2018;39(33):3021-104.

MACEDO ME., LIMA MJ., SILVA AO., ALCANTARA P., RAMALHINHO V., CARMONA J. Prevalência, Conhecimento, Tratamento e Controlo da Hipertensão em Portugal. Estudo PAP. Revista Portuguesa de Cardiologia, 2007;26(1):21-39.

CORTEZ-DIAS N., MARTINS S., BELO A., FIUZA M. Prevalência e Padrões de Tratamento da Hipertensão Arterial nos Cuidados de Saúde Primários em Portugal. Resultados do Estudo VALSIM. Revista Portuguesa de Cardiologia, 2009;28(5):499-523.

RIBEIRO AS., FARIA RJ. Prevalência de Factores de Risco Cardiovasculares e Lesão de Orgão-Alvo em Doentes com Hipertensão Arterial. Revista Portuguesa de Hipertensão e Risco Cardiovascular, 2013;33:4-7.

CORDINHÃ AC., PAÚL A., FERNANDES L. Obesidade Infantil e Hipertensão Arterial - a Realidade de uma População Pré-escolar. Acta Pediátrica Portuguesa, 2009;40:145-9.

PERDIGÃO C., ROCHA E., DUARTE JS., SANTOS A., MACEDO A. Prevalência, Caracterização e Distribuição dos Principais Fatores de Risco Cardiovasculares em Portugal. Uma Análise do Estudo AMÁLIA. Revista Portuguesa de Cardiologia, 2011;30(4):393-432.

UVA M., VICTORINO P., ROQUETTE R., MACHADO A., DIAS C. Investigação epidemiológica sobre prevalência e incidência de hipertensão arterial na população portuguesa - uma revisão de âmbito. Revista Portuguesa de Cardiologia, 2014;33(7-8):451-63.

INE. (2011). Resultados definitivos Census 2011. Lisboa, Portugal: INE, Instituto Nacional de Estatística.

MANCIA G., FAGARD R., NARKIEWICZ K., REDÓN J, ZANCHETTI A., BOHN J., et al. Guidelines for the management of arterial hypertension. Journal of Hypertension, 2013; 31(7): 1281-1357.

ROCHA E., MELLO E SILVA A., NOGUEIRA P. Hipertensão sistólica isolada: epidemiologia e impacto na prática clínica. Revista Portuguesa de Cardiologia, 2003;22(1):7-23.

PEREIRA M., AZEVEDO A., BARROS H. Determinants of awareness, treatment and control of hypertension in a Portuguese population. Revista Portuguesa de Cardiologia, 2010;29(12):1779-92.

GONZAGA C., SOUSA M., AMODEU C. Fisiopatologia da hipertensão sistólica isolada. Revista Brasileira de Hipertensão, 2009;16(1):10-4. 
ROSA E., FARIA A., LERÁRIO D. Obesidade, hipertensão arterial e suas influências sobre a massa e função do ventrículo esquerdo. Arquivos Brasileiros Endocrinologia e Metabologia, 2000;44(1):6471.

JORDAN J., YUMUK V., SCHALAICH M. Joint statement of the European Association for the Study ofObesity and the European Society of Hypertension: obesity and difficult to treat arterial hypertension. Journal of Hypertension, 2012;30(6):1047-55.

SILVA D, PETROSKI E., PERES M. Pré-hipertensão e hipertensão em adultos de Florianópolis: estudo de base populacional. Revista de Saúde Pública, 2012;46(6):988-98.

MACHADO H., ALVES A., TINOCO C., GONÇALVES C., MATOS C., REGO D. Prevalência do diagnóstico de hipertensão arterial em pressoas sedentárias e em praticantes de exercício físico, na cidade do Porto. Acta Médica Portuguesa, 2010;23:153-8.

KNOOPS K., GROOT L., KROMHOUT D., PERRIN A., MENOTTI A. Mediterranean Diet, Lifestyle Factors, and 10-Year Mortality in Elderly European Men and Women The HALE Project. The journal of the American Medical Association, 2004;292(12):1433-9.

ARITA N., MIYASHITA K., MORIOKA I., SHIRAISHI T. Antihypertensive Effects of Aerobic Exercise in Middle-Aged Normotensive Men with Exaggerated Blood Pressure Response to Exercise.

Hypertension Research, 2002;25:507-14s.

MONTEIRO M., SOBRAL D. Exercício físico e o controle da pressão arterial. Revista Brasileira de Medicina de Esporte, 2004;10(6):513-6.

FORJAZ C., TINUCCI T. A medida da pressão arterial no exercício. Revista Brasileira de Hipertensão, 2000;1:79-87.

RUIVO J., ALCANTARA P. Hipertensão arterial e exercício físico. Revista Portuguesa de Cardiologia, 2012;32(2):151-8.

GUS I., ZASLAVSKY C., MEDINA C., HARZHEIM E. Prevalência, Reconhecimento e Controle da Hipertensão Arterial Sistêmica no Estado do Rio Grande do Sul. Arquivos Brasileiros de Cardiologia, 2004;83(5):424-8. 


\section{ÍNDICE REMISSIVO}

\section{A}

Acidente Vascular Encefálico 26, 66, 67, 68, 70, 71, 72, 76

Acupuntura 23, 90, 91, 92, 93, 94, 95, 96

Aleitamento Materno 37, 38, 39, 40, 41, 42, 43, 44, 46, 47, 48, 50, 51, 52, 86, 89, 224, 225, 226, 227, 228, 229, 231

Alta Hospitalar 78, 79, 80, 81, 82, 84, 85, 86, 87, 88, 231, 289

Amputação 283, 284, 285, 286, 287, 288, 289, 293

Arboviroses 111, 112, 113, 114

Atenção Primária à Saúde 54, 55, 56, 62, 63, 111, 113

B

Barreira Lipídica 115

Biomarcador 30, 99, 100

Bisfosfonatos 15, 233, 234, 235, 236, 237, 238, 239, 240

C

Cuidado Farmacêutico 54, 55, 56, 57, 58, 61, 63

D

Diagnóstico Molecular 30

Doença Renal Crônica 16, 97, 98, 100, 107, 108, 109, 110, 262, 263, 264, 270, 271, 272

Dor $21,22,24,25,27,28,42,48,49,60,66,67,68,69,70,71,72,73,74,75,76,77,94$, $96,203,262,265,266,267,268,270,272,280,297$

E

Educação em Saúde 54, 57, 58, 60, 63, 78, 80, 89, 204, 230, 274, 275

Envelhecimento Cutâneo 158, 160

Éster 115, 116, 117, 118, 119, 121, 122, 123, 124, 125

Exercício Aeróbico 97, 101, 103

$\mathbf{F}$

Fibroblasto 126, 130, 135, 279

Fotoexposição 126, 127, 130, 131, 132, 133, 134, 135, 136

Função Renal 97, 99, 101, 102, 103, 104, 106, 107, 263, 268

G

Genograma 9, 10, 11, 13, 14, 16, 17, 18 
Gestação 41, 86, 144, 225, 226, 229, 273, 275

$\mathrm{H}$

Hanseníase 15, 9, 10, 11, 12, 13, 14, 16, 17, 18, 193, 194, 195, 196, 197, 198, 199, 200 , 201, 203, 204, 205

Hemodiálise 75, 263, 264, 267, 268, 269, 270, 271, 272

Hipertensão Arterial 61, 98, 99, 187, 241, 242, 243, 249, 250, 251, 252, 254, 259, 260, 261, 263

Hospitalização 1, 2, 6, 7, 25, 86, 106, 228, 230

I

Idoso 25, 68, 73, 76, 77, 160, 171

Interdisciplinaridade 1, 290, 293

M

Menopausa 15, 144, 233, 234, 235, 236, 237, 239

Micrornas 158, 159

Mortalidade Infantojuvenil 15, 208, 212

Multidisciplinaridade 1

$\mathbf{P}$

Parto 37, 38, 39, 40, 41, 42, 43, 44, 45, 46, 47, 48, 49, 50, 51, 52, 53, 78, 81, 144, 225, 228, 273, 274, 275

Práticas Integrativas 20, 21, 23, 24, 28, 29

Q

Qualidade de Vida 16, 3, 20, 21, 26, 27, 39, 60, 75, 77, 90, 95, 233, 234, 235, 262, 263, 264, 266, 267, 268, 269, 270, 271, 272, 274, 275, 277, 280, 295, 296, 297

$\mathbf{R}$

Reabilitação 26, 75, 77, 95, 204, 235, 283, 284, 285, 286, 287, 288, 289, 290, 291, 292, 293

Recém-Nascido 12, 37, 78, 79, 81, 82, 83, 84, 85, 86, 87, 88, 89, 224, 225, 226, 228, 231, 232, 274

Rejuvenescimento 158

$\mathbf{S}$

Sepse 181, 182, 183, 184, 185, 186, 187, 188, 189, 190, 191, 192

Sistema Auditivo 90, 91, 92, 94, 95 
Transtorno de Ansiedade 295, 302, 305

Transtorno de Humor 141, 142, 145

Transtorno Mental 235

$\mathrm{U}$

Úlcera Venosa 277

Unidade de Terapia Intensiva 78, 79, 80, 88, 89, 226 


\section{Condições Teórico-Práticas das Ciências da Saúde no Brasil 3}

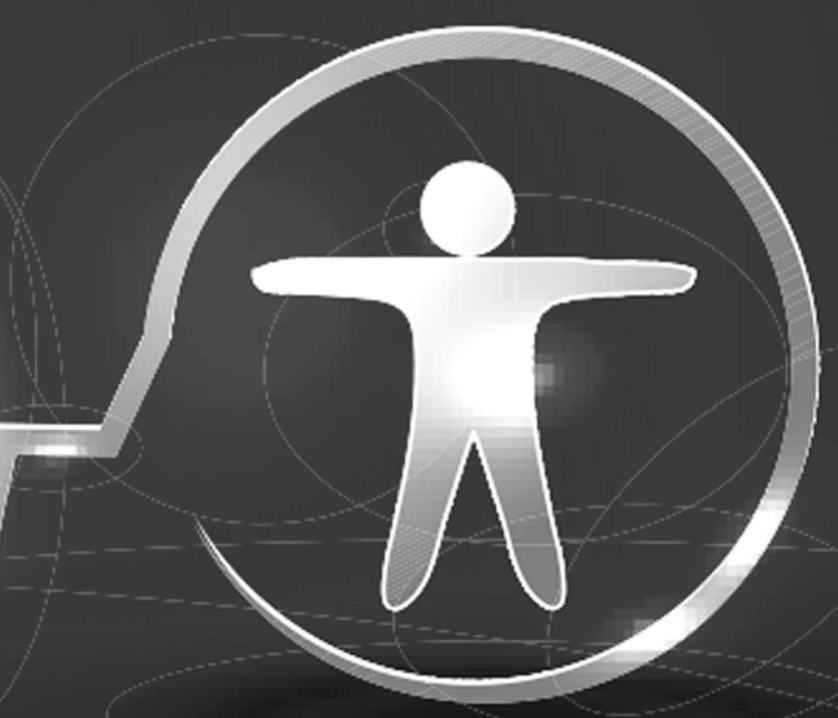

www.atenaeditora.com.br contato@atenaeditora.com.br \ @atenaeditora 0 www.facebook.com/atenaeditora.com.br if

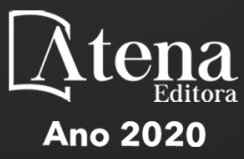




\section{Condições Teórico-Práticas das Ciências da Saúde no Brasil 3}

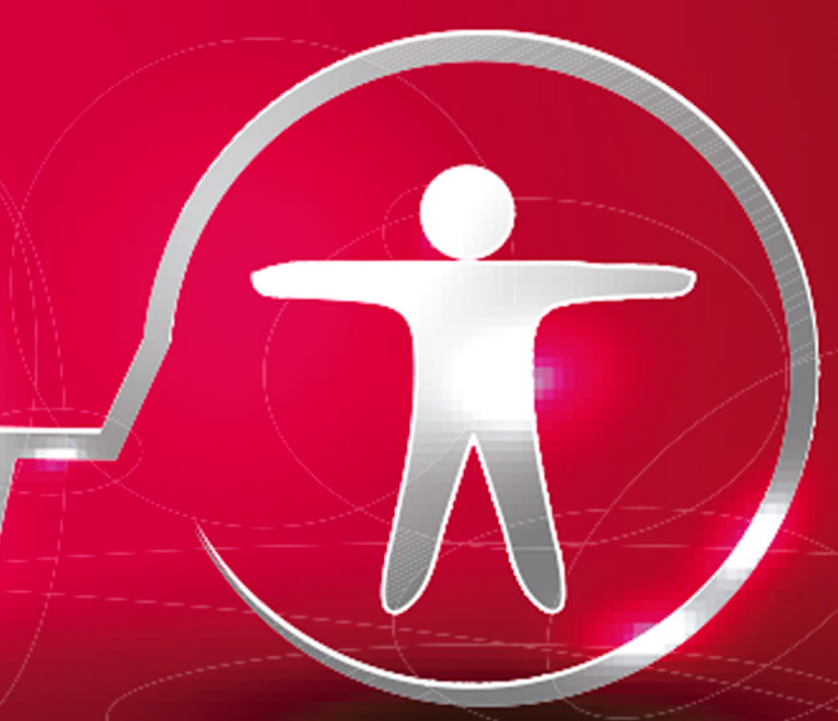

www.atenaeditora.com.br contato@atenaeditora.com.br \ @atenaeditora 우 www.facebook.com/atenaeditora.com.br if

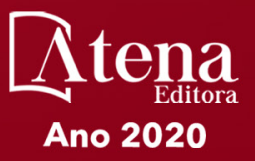

